\title{
Corticosteroid treatment for acute/ acute-on-chronic experimental and naturally occurring pancreatitis in several species: a scoping review to inform possible use in dogs
}

\author{
Kari-Anne Bjørnkjær-Nielsen ${ }^{1}$ and Charlotte Reinhard Bjørnvad ${ }^{2^{*}}$ (i)
}

\begin{abstract}
Acute pancreatitis in dogs is a prevalent disease characterised by mild to severe inflammation. Treatment with antiinflammatory corticosteroids has been widely debated but is not generally recommended in veterinary medicine. The objective of the present study was to present current evidence on the effect of corticosteroid treatment for acute/ acute-on-chronic pancreatitis across species. These findings were then used to evaluate if and how corticosteroid treatment could influence disease outcome in canine acute/acute-on-chronic pancreatitis. A scoping review was performed by searching the Agricola, CAB Abstracts, MEDLINE and Embase databases to identify relevant articles published before June 24, 2021. The inclusion criteria were English language, original research published in a peerreviewed journal, and investigation of corticosteroid treatment effects on acute/acute-on-chronic pancreatitis by the outcome parameters clinical score, circulating CRP level, hospitalisation duration, mortality and pancreas histopathology. Research on any species was considered. Studies were rated based on the level of evidence, and methodological quality was evaluated based on similarity between groups at baseline, risk of bias and study group size. The reporting method was based on the PRISMA extension for scoping reviews. One thousand nine hundred fifty-four studies were identified, and 31 met the inclusion criteria. Five were canine studies, with 4 investigating experimentally induced pancreatitis; 5 were human clinical studies; and 21 were rodent studies of experimentally induced pancreatitis. The level of evidence ranged between randomised controlled trials and case series, the estimated risk of bias ranged from low to high, and the sample sizes ranged from very small to moderate. Evidence indicates that adding corticosteroid to symptomatic treatment of acute/acute-on-chronic canine pancreatitis could have a positive influence on disease outcome. However, the analysed evidence was based on several species, including both naturally occurring and experimentally induced pancreatitis; thus, the authors suggest that large randomised controlled studies should be performed in dogs with spontaneously occurring acute/acute-on-chronic pancreatitis to further elucidate a potential benefit of corticosteroid treatment.
\end{abstract}

Keywords: Canine, Dexamethasone, Glucocorticoid, Hydrocortisone, Steroid

\footnotetext{
*Correspondence: crb@sund.ku.dk

${ }^{2}$ Department of Veterinary Clinical Sciences, Faculty of Health

and Medical Sciences, University of Copenhagen, Dyrlægevej 16, 1870 Frederiksberg C, Denmark

Full list of author information is available at the end of the article
}

\section{Background}

Canine acute pancreatitis is a common disease in veterinary practice, and the clinical presentation can vary from subclinical or mild nonspecific clinical signs to severe life-threatening disease [1].

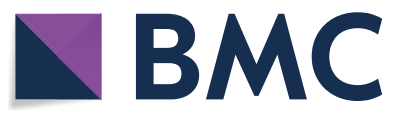

(c) The Author(s) 2021. This article is licensed under a Creative Commons Attribution 4.0 International License, which permits use, sharing, adaptation, distribution and reproduction in any medium or format, as long as you give appropriate credit to the original author(s) and the source, provide a link to the Creative Commons licence, and indicate if changes were made. The images or other third party material in this article are included in the article's Creative Commons licence, unless indicated otherwise in a credit line to the material. If material is not included in the article's Creative Commons licence and your intended use is not permitted by statutory regulation or exceeds the permitted use, you will need to obtain permission directly from the copyright holder. To view a copy of this licence, visit http://creativeco mmons.org/licenses/by/4.0/. The Creative Commons Public Domain Dedication waiver (http://creativecommons.org/publicdomain/ zero/1.0/) applies to the data made available in this article, unless otherwise stated in a credit line to the data. 
Our understanding of the pathophysiology of acute pancreatitis is largely extrapolated from human clinical studies and experimental animal models. An apical block is believed to cause intracellular fusion of zymogen granules and lysosomes, which leads to the activation of trypsinogen and release of cathepsin-B and other pancreatic enzymes within acinar cells. These changes activate the apoptotic cascade and cause inflammation, which is manifested by neutrophil migration to the pancreas as well as probable complement activation and a "cytokine storm" that further contributes to inflammation [2,3].

The fact that pancreatitis is an inflammatory condition is supported by increases in parameters associated with inflammation, such as C-reactive protein (CRP), Interleukin 6 (IL-6) and Tumor necrosis factor $\alpha$ (TNF- $\alpha$ ) [4], as well as histologic findings [5]. Although universally standardised criteria are not available for the histologic classification of pancreatitis in dogs [6, 7], canine acute pancreatitis has been defined by Watson et al. as pancreatic necrosis that frequently presents with a neutrophilic infiltrate but without fibrosis or chronic inflammation [8]. This definition is consistent with Newman et al. [9] who suggest that neutrophilic inflammation is often associated with pancreatic necrosis and/or peripancreatic fat necrosis. These findings are considered potentially completely reversible. The presence of permanent histopathological changes, such as fibrosis and acinar atrophy, suggest chronic pancreatitis, and a mononuclear or mixed inflammatory infiltrate is expected [8-10].

In the clinical setting, distinguishing between acute and chronic canine pancreatitis is difficult. Truly acute disease cannot be distinguished from acute-on-chronic pancreatitis [11], and histopathological assessments are infrequent due to the cost and/or possible associated morbidity [7, 12, 13]. Furthermore, studies show that in many histopathological samples, acute and chronic changes are found concurrently $[10,14]$, which make the distinction between acute and chronic pancreatitis less relevant. This mix of acute and chronic disease has led authors to conclude that chronic pancreatitis may result from recurrent acute disease [7] and that some apparently acute pancreatitis cases are in fact acute exacerbations of previously unrecognised chronic disease [11].

Therefore, this study includes cases of acute-onchronic as well as truly acute canine pancreatitis, which is referred to as CAP, when discussing the condition in dogs with acute symptoms of pancreatitis.

Canine acute pancreatitis can result in acute lifethreatening systemic complications, such as disseminated intravascular coagulation and multiple organ failure [2]. Ongoing inflammation can result in progressive fibrosis and loss of exocrine and/or endocrine tissue, thus causing the development of exocrine pancreatic insufficiency and/or diabetes mellitus [2, 11]. Hence, initiating the optimal treatment for CAP as soon as possible can have considerable positive results.

Although multiple risk factors and rare underlying causes have been identified [1,7], most cases of CAP are considered idiopathic and do not present an underlying disease for treatment [6]. The mainstay treatment recommended for CAP includes aggressive fluid therapy, a low-fat diet, antiemetics and analgesic agents, including opioids $[15,16]$.

As a confirmed inflammatory disease, it is noteworthy that anti-inflammatory medications are rarely discussed for the treatment of CAP. A search of the literature shows that although nonsteroidal anti-inflammatory agents (NSAIDs) are generally not recommended [15-20], a consensus has not been reached regarding the use of corticosteroids. Corticosteroids are generally contraindicated because of the risk of gastric ulcerations and reduced reticuloendothelial activity [19]. Recent human case studies have indicated that corticosteroids may even induce acute pancreatitis [21, 22]. In dogs with hyperadrenocorticism, one study reported increases in canine pancreatic-specific lipase (Spec-cPL) concentrations without any obvious clinical evidence of pancreatitis [23]. However, dogs in this study were not evaluated for evidence of subclinical pancreatitis. In another study, hyperadrenocorticism resulted in an increased prevalence of ultrasonographic pancreatic hyperechogenicity compared with normal dogs, despite that they had normal Spec cPL concentrations and no obvious signs of clinical pancreatitis [24]. It should also be noted that hyperadrenocorticism has been identified as a risk factor for developing fatal acute pancreatitis [25]. However, a study in 2018 on healthy beagles showed that even immunosuppressive doses of prednisolone did not induce clinical or histological evidence of pancreatitis [26]. Several authors have indicated the lack of evidence suggesting that steroids reduce pancreatic inflammation [19], and some have narrowed this statement to apply to dogs only [20]. In contrast, a recent publication suggested that morbidity may be improved and/or mortality may be reduced in CAP by using low-dose corticosteroids [16].

This study performed a scoping review of peerreviewed literature to present current evidence on the effects of corticosteroid treatment on acute/acute-onchronic pancreatitis across species to provide recommendations regarding the use of corticosteroids for CAP.

\section{Search strategy Methods}

The method of reporting was performed according to the Preferred Reporting Items for Systematic Reviews and Meta-Analyses (PRISMA) extension for scoping 
reviews [27]. A review protocol including inclusion and exclusion criteria for the screening processes as well as a search strategy was defined prior to starting this review; this protocol was dated May 12, 2019 and is available on request. The clinical research question, which was formulated by the PICO method [28], was as follows: In dogs with acute/acute-on-chronic pancreatitis receiving standard treatment, could adjuvant therapy with corticosteroids improve the disease outcome relative to that of standard treatment alone? Markers of improved disease outcome were clinical score (as defined by the authors), circulating CRP levels, hospitalisation duration, mortality rate and pancreas histopathology.

\section{Eligibility criteria \\ Types of studies}

Studies reporting how corticosteroid treatment affects acute/acute-on-chronic pancreatitis with regard to at least one of the abovementioned markers of disease outcome were considered. As a sparse amount of literature was expected, studies were not excluded based on the study design or restricted by publication date; however, the language was restricted to English. Articles for which full text was not available or that were not peer-reviewed according to the publisher's webpage or the article's cover page were excluded. Conference abstracts were excluded since they are frequently publicly unavailable. Reviews and meta-analyses were excluded because the relevant original studies were included when relevant and available instead.

\section{Types of participants}

As few studies in dogs were expected, exclusions based on species were not performed. Individuals suffering from acute/acute-on-chronic pancreatitis that spontaneously occurred or was experimentally induced were included. In vitro studies were excluded. Human studies on autoimmune pancreatitis were excluded because this disease has not been documented to cause acute pancreatitis in dogs [7]. Human paediatric studies were excluded because a strong genetic background is suspected [29], which is unknown in dogs. Human studies in which acute pancreatitis was found to be a sequela to or part of another specific disease (e.g., systemic lupus erythaematosus) or treatment thereof (medical or surgical) were excluded. Concomitant biliary disease and alcoholism in humans were accepted because they are considered predisposing factors.

\section{Types of intervention}

Studies reporting corticosteroid treatment as a single treatment or an adjuvant to standard treatment of acute/acute-on-chronic pancreatitis were considered.
No exclusion due to corticosteroid type or dose was conducted. However, studies involving anabolic steroids as well as endogenous corticosteroids were excluded. Studies reporting the preventive effect of corticosteroids given prior to or simultaneously with inducing experimental acute pancreatitis and studies reporting corticosteroid treatment as a cause of pancreatitis were excluded.

\section{Types of comparison/control}

Individuals with acute/acute-on-chronic pancreatitis following the same supportive treatment protocol apart from corticosteroid treatment were used as model controls.

\section{Types of outcome}

The primary outcome was the corticosteroid treatment effect based on the clinical score (as defined by the authors), circulating CRP levels, hospitalisation duration, mortality and pancreas histopathologic evaluation.

\section{Information sources}

A literature search was conducted by the first author (KBN) from August 21 to August 28, 2019, using the following databases: Agricola (1970-present), CAB Abstracts (1910-present), MEDLINE (1946-present), and Embase (1974-present). No limits were applied to publication year.

\section{Search}

The following search terms were queried in the electronic databases: Steroid"; corticosteroid"; cortiso"; glucocorticoid"; dexamethason"; hydrocortiso*; predniso*; methylpredniso"; acute pancreatiti"; acute on chronic pancreatiti*; acute-on-chronic pancreatiti*. For the complete search strategy, see Additional file 1.

\section{Study selection}

The eligibility assessment was based on titles and abstracts in an unblinded standardised manner. Studies fulfilling the inclusion criteria and those in which criteria fulfilment could not be determined by title or abstract were retrieved as full texts and assessed by the same criteria. References of included articles as well as publications reviewing corticosteroid treatment for CAP/acute/ acute-on-chronic pancreatitis from 2010 onward were screened manually for additional articles of relevance. Duplicates were manually removed. A Prisma 2009 flow diagram was used to elucidate the study selection process (Fig. 1).

\section{Data collection process}

A data charting form (Table 1) was developed using Microsoft Word 2010 software to extract relevant 


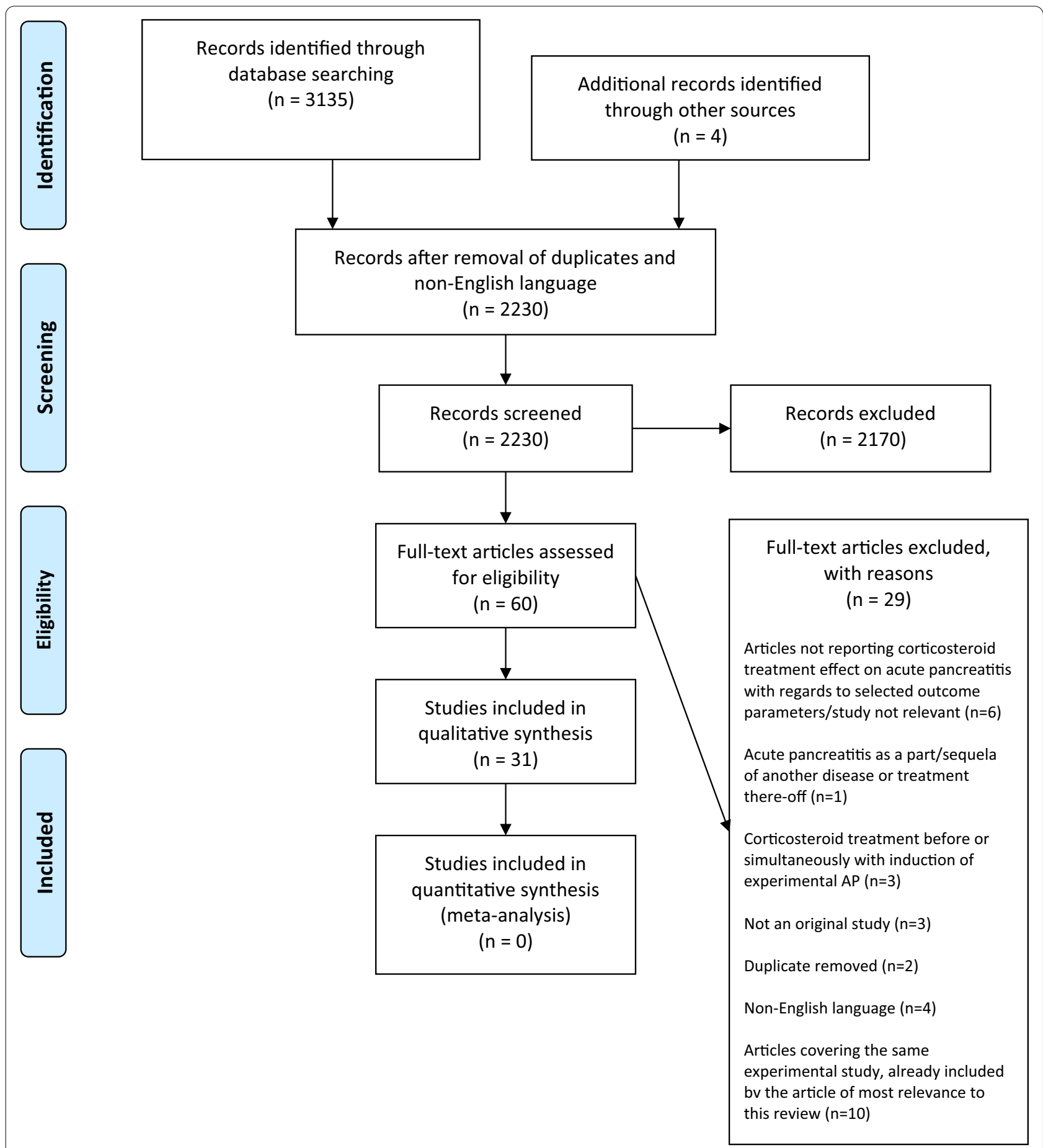

Fig. 1 PRISMA 2009 flow diagram elucidating the inclusion and exclusion process for studies found in the database search. From Moher et al. [89]

information from the included studies regarding the study protocol and the results of the corticosteroid treatment effect. Ten randomly chosen studies were used to test the form and adjust the design.

\section{Data item}

The following information was assessed or directly extracted from each included study: (1) Level of evidence, study design and methodological quality; (2) 
Species studied; (3) Number of individuals in corticosteroid-treated groups (GC groups) and model control groups; (4) Type of acute pancreatitis (AP) (spontaneous or experimentally induced); (5) Corticosteroid treatment; (6) Other treatments; (7) Data collection and timing; (8) Outcome: Clinical score (as defined by the authors); (9) Outcome: CRP level; (10) Outcome: Hospitalisation duration; (11) Outcome: Mortality rate; and (12) Outcome: Pancreas histopathology score.

\section{Critical appraisal of individual sources of evidence}

Both authors individually assessed the level of evidence and quality of the method for all included studies. In case of discrepancies, a consensus was reached through discussion.

The individual studies were graded for level of evidence (LOE) on a scale of I to IV [30]. LOE I studies included relevant primary studies (systematic reviews and metaanalyses were originally included but were ultimately excluded) (Fig. 1); LOE II studies included randomised controlled trials (RCTs); LOE III studies included nonrandomised controlled trials and retrospective case-control series; and LOE IV studies included case series and represented the lowest LOE.

The quality of the method of the individual studies was evaluated for the following 3 components: group similarity, risk of bias, and study group sizes.

Group similarity was rated good, fair or poor according to the description of health/disease status, age (or weight of rodents), sex and breed (dog studies only) of study subjects. When experimental animals were used, no description of disease was interpreted to mean that the animals were healthy.

Good group similarity: All healthy before induction of pancreatitis, or acute pancreatitis (AP) diagnoses for all participants based on clinical signs compatible with $\mathrm{AP}$ and at least one of the following: Positive cPLI (Spec cPL) or DGGR lipase, diagnostic imaging (ultrasound or CT) findings indicative of AP, histopathological findings indicative of AP, and similar age, sex and breed characteristics.

Fair: All healthy or AP diagnosis for all participants based on at least clinical signs compatible with AP and increased plasma lipase/amylase, no obvious skewing of group participants regarding age, sex and breed.

Poor: Information on diagnostic criteria for AP diagnosis is lacking or information regarding age/weight, breed or sex of study subjects is lacking.

The risk of bias of individual studies was graded as high, moderate or low according to the evaluated risk of selection, performance, detection, attrition, reporting and other bias. The evaluation was performed using the criteria set up by the Cochrane Collaboration's tool for assessment of risk of bias [31].

Study group size was graded as good, moderate, small or very small according to criteria used in other veterinary systematic reviews: $>50$ (good), 20-49 (moderate), 10-19 (small), <10 (very small) [32-35]. The collected information is presented in Table 2.

\section{Synthesis of results}

The outcome parameters for evaluating the effect of corticosteroid treatment on acute pancreatitis were the clinical score (as defined by the authors), circulating CRP levels, hospitalisation duration, mortality rate and pancreas histopathology score. Among the included studies, evidence for the corticosteroid treatment effect for each outcome parameter was collected and summarised narratively. The evidence for each outcome parameter was graded good, fair or insufficient, according to the following criteria modified from Jensen and Bjørnvad [35]:

Good evidence: Multiple RCTs with a low risk of bias or with a moderate risk of bias and a moderate to good study size.

Fair evidence: At least 1 RCT had a low risk of bias or a moderate risk of bias and a moderate to good study size.

Insufficient evidence: No RCTs had a low risk of bias or a moderate risk of bias and moderate to good study size.

Good or fair evidence for positive effect of corticosteroid treatment would indicate support of corticosteroid treatment. Alternatively, good or fair evidence for lack of effect or adverse effects would result in the advice against corticosteroid treatment.

\section{Ethical considerations}

In 20 of the 21 experimental rodent studies, the study protocols were documented to have followed national ethical guidelines and/or had been approved by an ethical committee; the exception was Wang et al. [36]. No ethical considerations were mentioned in the canine studies. Two human studies were approved by an ethical committee $[37,38]$.

\section{Review}

\section{Study selection}

After removing duplicates and non-English language articles, a total of 2230 citations were identified by electronic databases and manual searches of article references. Screening of titles and abstracts excluded 2170 of these citations. Sixty full-text articles were assessed for eligibility, and 29 were excluded for reasons stated in the PRISMA flow diagram (Fig. 1). A reference list of the 29 excluded studies is available on request. Finally, 31 studies were included in this review. 


\section{Study characteristics}

Of the 31 studies included in the review, 5 involved dogs, 5 involved humans and 21 involved rodents (Table 1).

Regarding the study design, 20 studies were categorised as RCTs (Tables 1 and 2); of these, 1 was a canine study [39], 2 were human studies [38, 40] and 17 were rodent studies [36, 41-56]. Only 1 study [38] described the method of randomisation. Eight studies were categorised as nonrandomised controlled trials, with 4 involving canines [57-60] and 4 involving rodents [61-64]. Two human studies were categorised as retrospective case control studies $[37,65]$, and 1 human study was categorised as a case series [66] (Tables 1 and 2).

The studies involved mainly very small to moderate size study groups ranging between 4 and 45 participants (dogs, humans and rodents). One control group had 53 participants [40] (Table 2).

One canine study concerned dogs with spontaneously occurring AP [57], and 4 studies concerned healthy dogs with experimentally induced AP [39, 58-60]. The 5 human studies all concerned patients with spontaneously occurring AP receiving treatment in hospital settings (Table 1). All 21 rodent studies concerned experimentally induced AP. Methods of inducing experimental AP varied among the studies (Table 1).

All subjects in the included studies were provided similar supportive treatment. Dogs suffering from spontaneous AP were treated according to standard protocols, including IV fluids, antibiotics, opioid analgesics, antiemetics, antacids and vitamin supplements [57]. Human subjects were treated with the previously mentioned supportive treatments as well as anticholinergics, nasogastric suction, pancreatin secretion inhibition, parenteral nutrition, oxygen, dextran 40, Chinese Dachengqi decoction, and blood plasma and albumin transfusion [37, 38, 40, 65, 66]. Rodents and dogs subjected to induced AP were generally not treated or only treated with IV or SC fluids. Few were treated with analgesics $[47,62]$ or antimicrobials $[58,64]$. In addition, test subjects (GC groups) were given corticosteroids while model controls were not.

Different types of corticosteroids were used in the included studies. Seven studies used hydrocortisone, including 2 dog studies [39, 60], 2 human studies [37, 65] and 3 rodent studies [43, 44,61]. Prednisolone was used in 1 canine study [57] and 2 rodent studies [44, 50], while methylprednisolone was used in 6 rodent studies [42, $46,51,52,55,63]$. Cortisone acetate was used in just 1 canine study [59], whereas dexamethasone was used in 1 canine study [58], 3 human studies [38, 40, 66] and 10 rodent studies [36, 41, 45, 47-49, 54, 56, 62, 64]. The type of corticosteroid used for treatment was not specified in one of the rodent studies [53]. The dose, administration route and start time of corticosteroid treatment varied widely among studies (Table 1).

Of the outcome parameters, the clinical score was reported in 4 studies. In dogs, Attix et al. [58] subjectively evaluated the activity and general appearance by clinical examination while Okanishi et al. [57] scored patients 0-3 on specific parameters (weakness/lethargy, appetite, vomiting, stool condition, abdominal pain). In the two human studies, the clinical outcome evaluation method was not described $[65,66]$.

Circulating CRP was measured in 5 studies, namely, 1 canine [57], 1 human [37] and 3 rodent studies [44, 51, 52]; however, for 2 of the rodent studies [51, 52], the results were not presented.

The duration of hospitalisation was reported in 1 canine study [57] and 1 human study [38].

Mortality rates were reported in 18 studies, including 4 canine $[39,57,59,60]$, all 5 human studies $[37,38,40,65$, 66] and 9 rodent studies [36, 41, 42, 48, 50, 53, 61, 63, 64].

Histopathological examination of the pancreas was performed in 23 studies, including 3 canine and 20 rodent studies (Table 1). A histopathological scoring system was used in 17 of these 23 studies. Oedema, inflammatory infiltration, necrosis and haemorrhage was frequently rated using a score of $0-3$ or $0-4$, sometimes, fat necrosis or vacuolisation was rated in a similar way. In 6 studies, the histopathological scoring technique was not reported [48, 49, 56, 58, 59, 64]. Ten studies did not present histopathological results $[36,49,51,52,56,58$, $59,61,64]$; of these, 7 studies reported conclusions that were not statistically evaluated $[45,49,51,52,56,59,61]$ while 1 reported statistically significant conclusions [36]. In one study the results were considered unclear [43].

\section{Risk of bias within studies}

An overview of the "risk of bias" for the individual studies can be found in Table 2. In general, a great variance in risk of bias was found among the studies, and it did not seem to depend on time since publication.

For studies concerning spontaneously occurring AP, the estimated risk of bias was categorised as low for 2 human studies [37, 38], moderate for 1 canine study [57] and 1 human study [40], and high for 2 human studies $[65,66]$. Study group sizes were found to be moderate in 1 canine study [57] and 3 human studies [38, 40, 66] and small in 2 human studies [37, 65]. Similarity at baseline was poorly characterised in 1 human study [40], fair in 2 human studies $[65,66]$ and good in 1 canine study [57] and 2 human studies [37, 38].

For studies concerning dogs with experimentally induced AP, the estimated risk of bias was categorised as low for 2 studies [39, 60] and high for 2 studies $[58,59]$. Study group sizes were small or very small, and similarity 
at baseline was poorly characterised for all 4 studies [39, $58-60]$.

Studies concerning rodents with experimentally induced AP varied in risk of bias. A low risk of bias was found in 8 studies [42, 44, 46, 47, 53-55, 61]. Six studies were categorised as moderate [36, 41, 43, 50, 62, 63], and 7 studies were categorised as high $[45,48,49,51,52$, 56, 64]. Regarding group size, 6 studies were categorised as very small $[46,51,52,55,56,62]$, 7 studies were categorised as small [42-44, 49, 50, 53, 64], 7 studies were categorised as moderate $[36,41,45,48,54,61,63]$ and 1 study did not provide group size information [47]. With regard to similarity at baseline, all studies except for 1 were categorised as well characterised, and the exception was categorised as fair [53].

No studies had sample size calculations performed prior to the start of the study.

Few studies mentioned drop-outs. Okanishi et al. [57] had 3 drop-outs, which they excluded from relevant analyses without commenting on possible implications regarding the results. No other canine studies mentioned drop-outs. In human studies, only Wan et al. mentioned that statistically significant differences were not observed in the experimental groups following drop-outs, and they clearly described the drop-out cases [38]. In the rodent studies, one study chose to exclude from the histopathological analysis rodents that died during the experiment; numbers were not presented and possible implications for the results were not described [50]. The rest of the studies did not report drop-outs.

Several studies did not include statistical analyses. Among the human studies, 2 provided clinical score and mortality conclusions without presenting statistical analyses $[65,66]$. Two canine studies did not present statistical evaluations of their conclusions on clinical score [58], mortality and histopathology [59]. Six rodent studies did not statistically evaluate histopathological outcomes [45, $49,51,52,56,61]$. Two rodent studies presented conclusions on mortality without statistical analyses [63, 64].

\section{Results of the individual studies and synthesis of results}

For an overview of the results of individual studies, please refer to Tables 1 and 2.

Clinical score results were reported in 4 studies. One canine study on spontaneous AP reported a significantly shortened time (median 4 versus 7 days) to reach a clinical severity score of $\leq 2 / 3$ compared to the model controls [57]. Three other studies (1 canine and 2 human studies) reported results on clinical scores without statistically evaluating the data $[58,65,66]$.

Regarding the effect of corticosteroids on circulating CRP levels, one rodent study [44] found that hydrocortisone or prednisolone administered $1 \mathrm{~h}$ after induction of experimental AP resulted in slight but statistically significant decreases in CRP levels $(16.8 \pm 1.4$ and $19.2 \pm 1.6 \mathrm{mg} / \mathrm{dL}$, respectively) compared with the no-steroid treatment $(25.6 \pm 1.7 \mathrm{mg} / \mathrm{dL}, \mathrm{P}<0.05)$, while the same treatment administered $3 \mathrm{~h}$ later did not have these effects $(24.3 \pm 1.6$ and $24.8 \pm 1.8 \mathrm{mg} / \mathrm{dL}$, respectively, Table 1). For spontaneously occurring AP in dogs, Okanishi et al. found that the time for CRP levels to reach $<2 \mathrm{mg} / \mathrm{dL}$ was significantly shorter in prednisolone-treated dogs than in model controls [57]. Eklund et al. [37] found that CRP levels were significantly lower in hydrocortisone-treated human patients at $48 \mathrm{~h}$ after starting treatment but not at $24 \mathrm{~h}$ relative to standard (non-corticosteroid)-treated patients (Table 1).

The duration of hospitalisation was evaluated in 1 canine and 1 human study [38, 57]. Both studies found that the duration of hospitalisation was significantly reduced (median 5 vs 8 days and 32.5 vs 49.2 days) in corticosteroid-treated patients compared to non-corticosteroid-treated patients (Table 1).

Eleven studies provided statistically evaluated results on the mortality rate (Table 1). Seven studies found a statistically significant improvement in survival or reduction in mortality. Four studies found no statistically significant difference in mortality/survival. In dogs with spontaneously occurring AP, Okanishi et al. [57] found that 1 -month survival was significantly higher $(88.7 \%$ vs $57.9 \%$ ) in prednisolone-treated dogs than in dogs receiving no corticosteroids (Table 1). In humans, Liu et al. found significantly reduced mortality in dexamethasonetreated patients compared to non-corticosteroid-treated patients (Table 1) [40]. However, this study was confounded by the fact that the corticosteroid group was also treated with dextran and Salvia miltiorrhiza. Wan et al. [38] found no significant difference in mortality between dexamethasone-treated and standard-treated human patients (Table 1).

In canine studies of experimentally induced AP, two studies reported significantly increased survival time (Table 1). Studley and Schenk [39] administered lowdose hydrocortisone 6 or $12 \mathrm{~h}$ after induction of AP and administered hydrocortisone at low or high doses $6 \mathrm{~h}$ after induction, and both of these studies showed a significantly increased survival time with hydrocortisone treatment compared to IV fluids only, regardless of the dose or timing of treatment. Imahori et al. [60] administered a similar low dose of hydrocortisone $6 \mathrm{~h}$ after induction and found that survival time was significantly increased in the hydrocortisone-treated group compared to the non-corticosteroid-treated group.

Six rodent studies statistically evaluated the corticosteroid effects on mortality in experimentally induced AP (Table 1). Three studies found significantly improved 


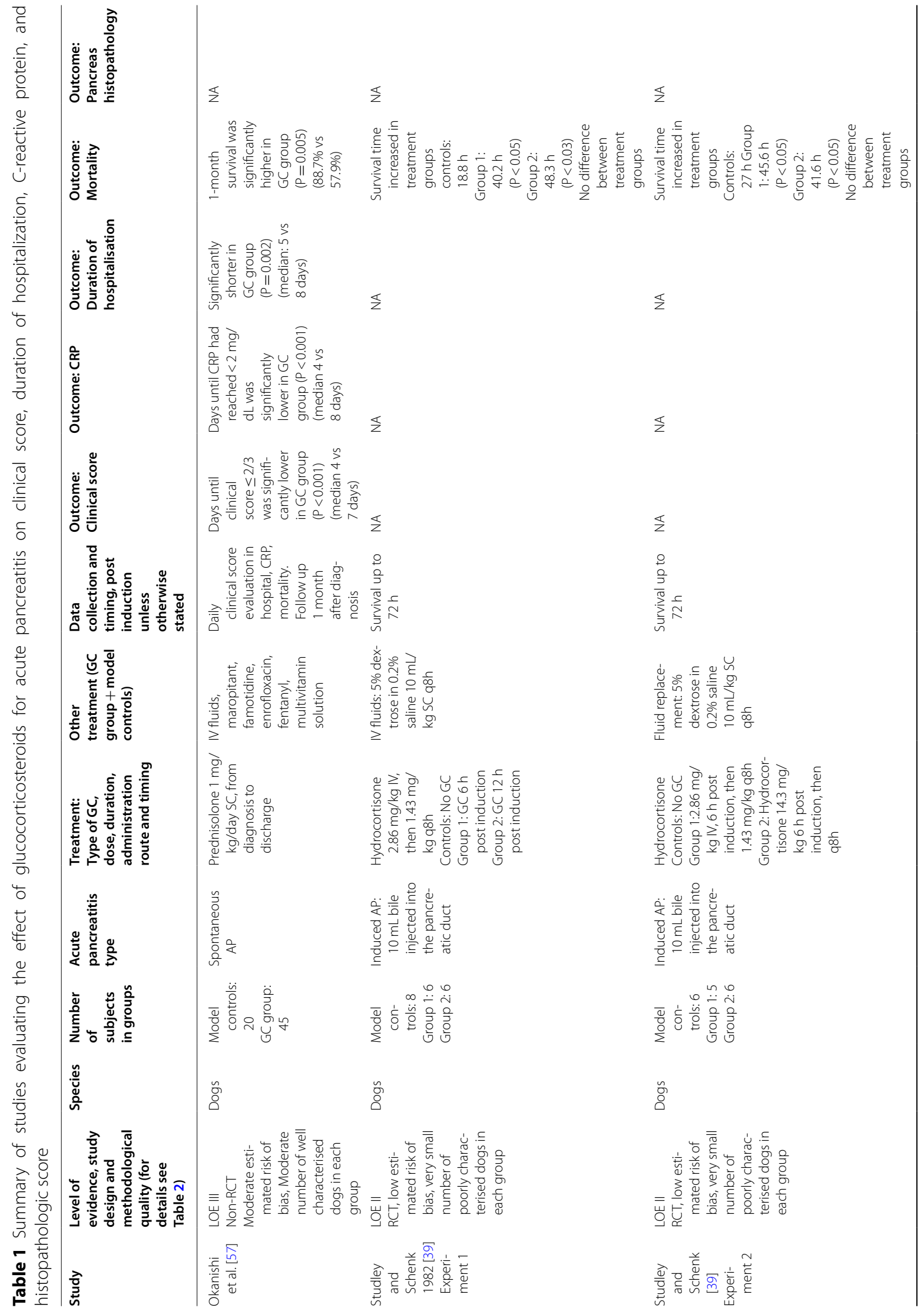




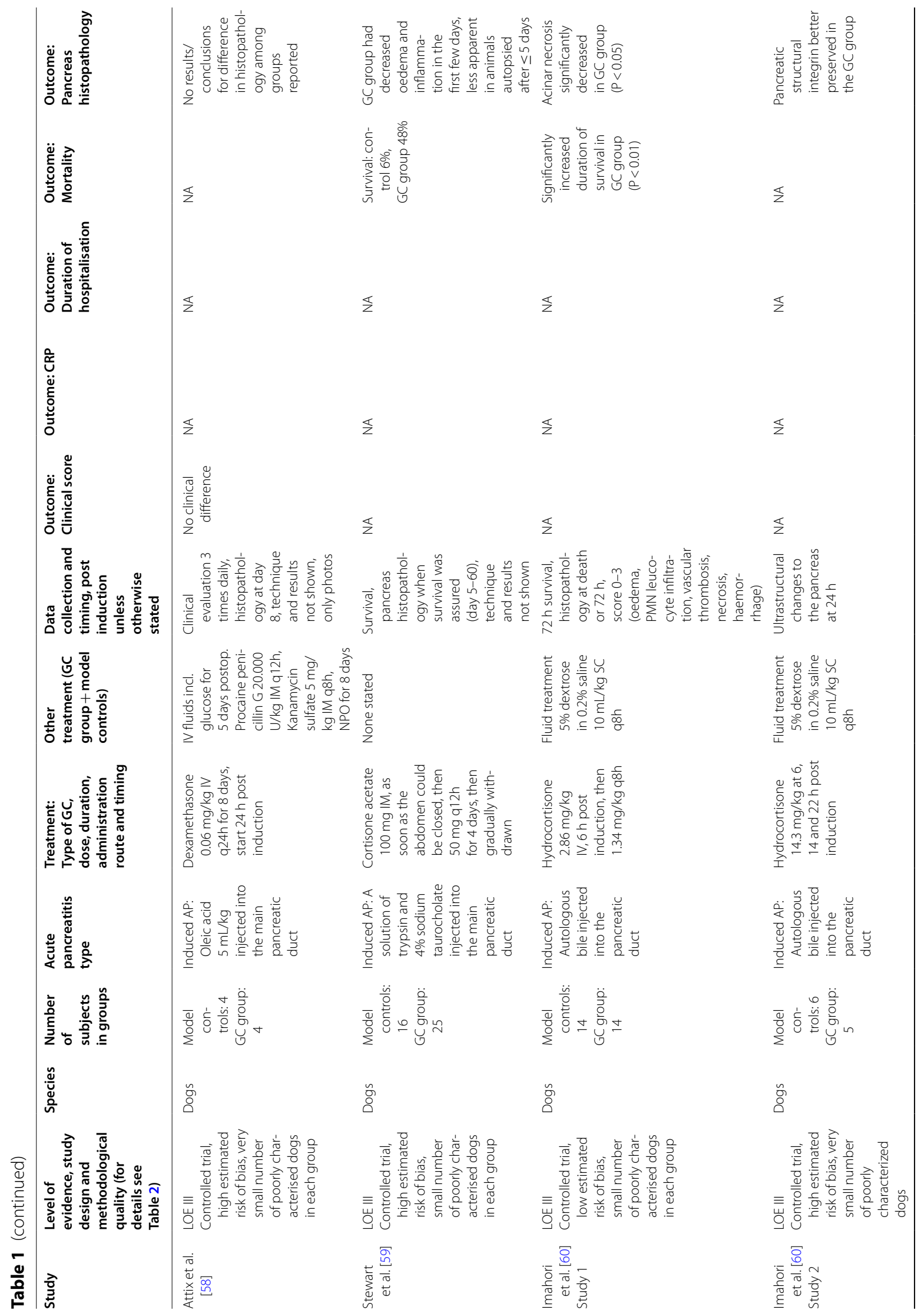




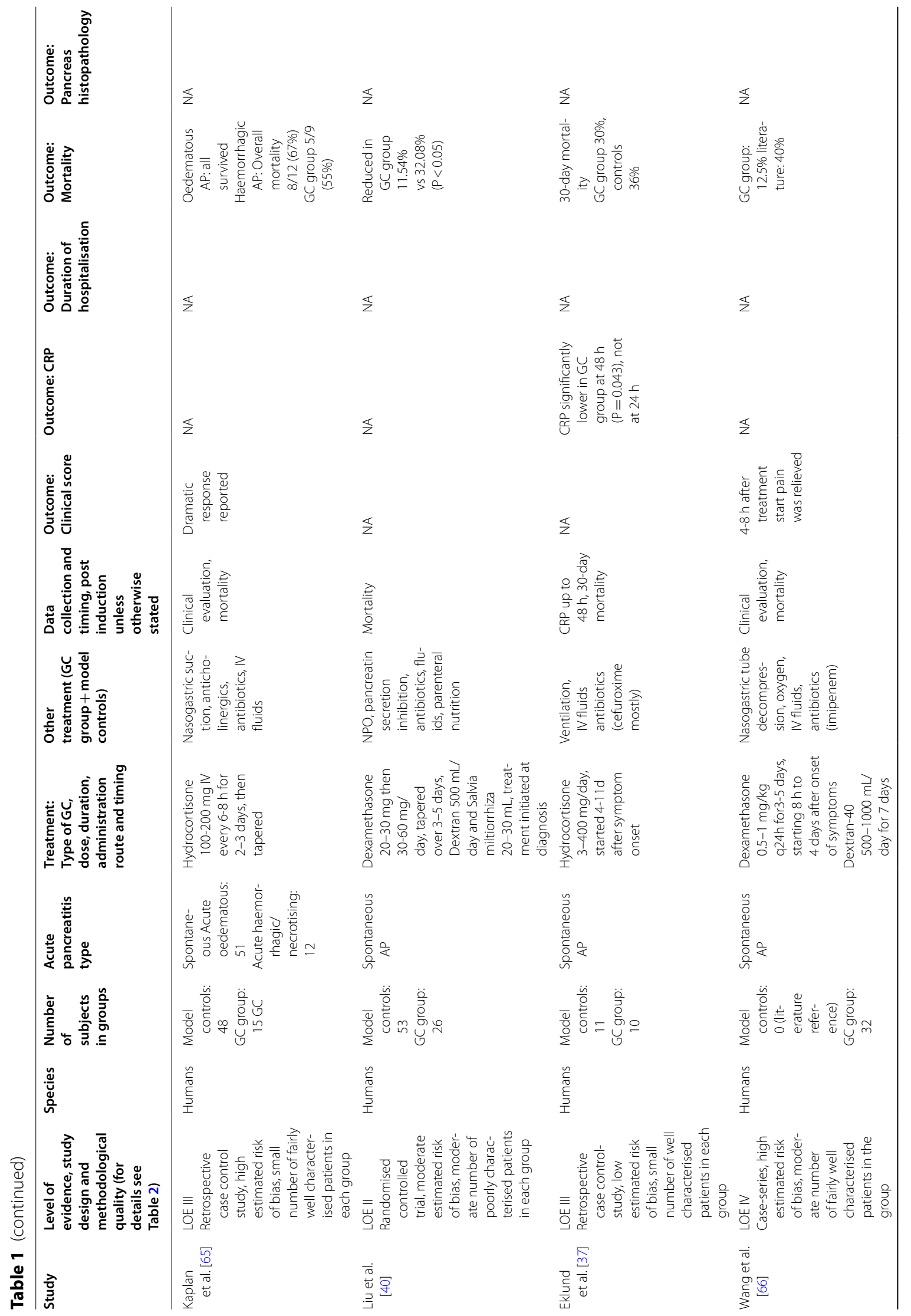




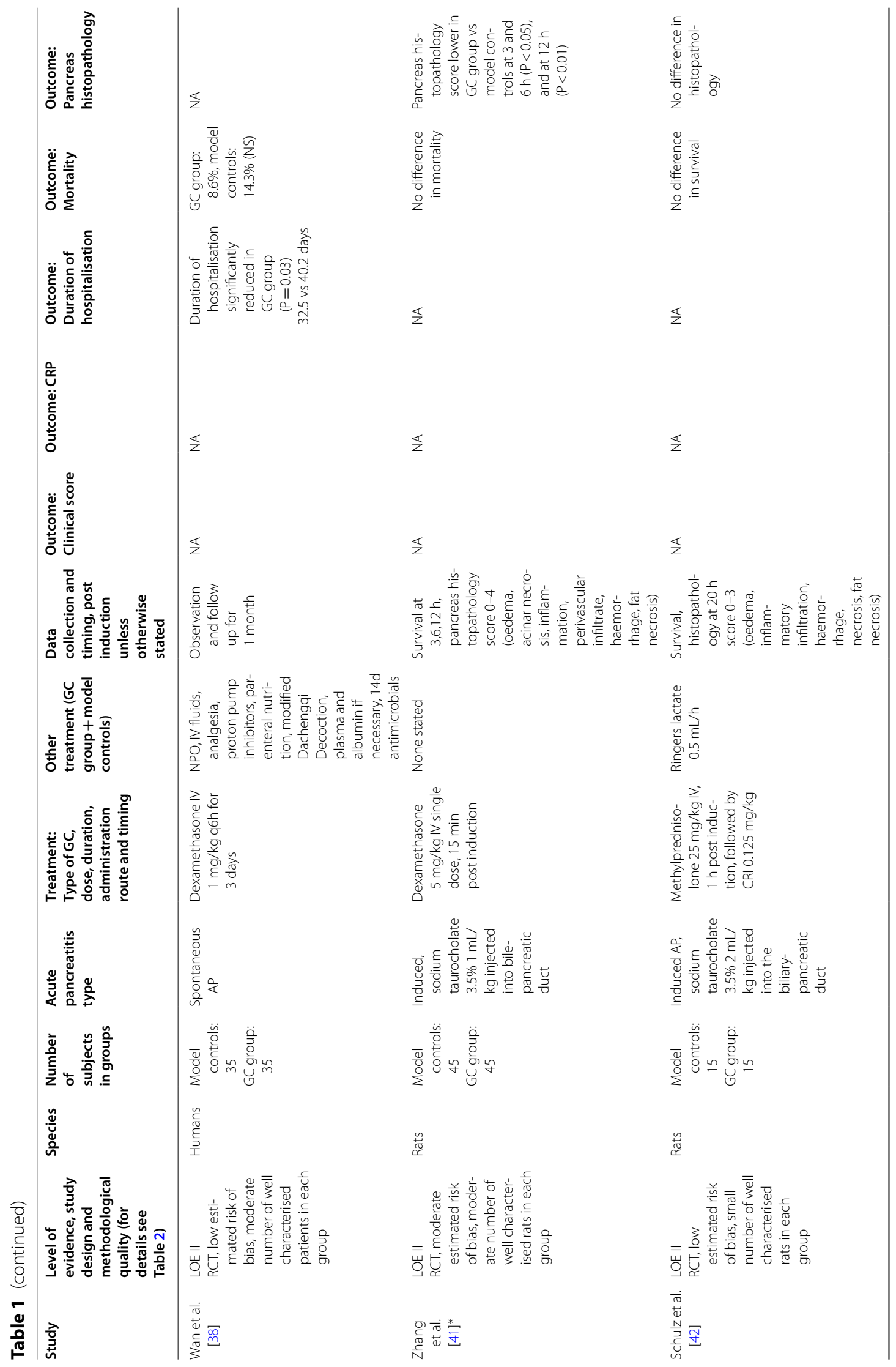




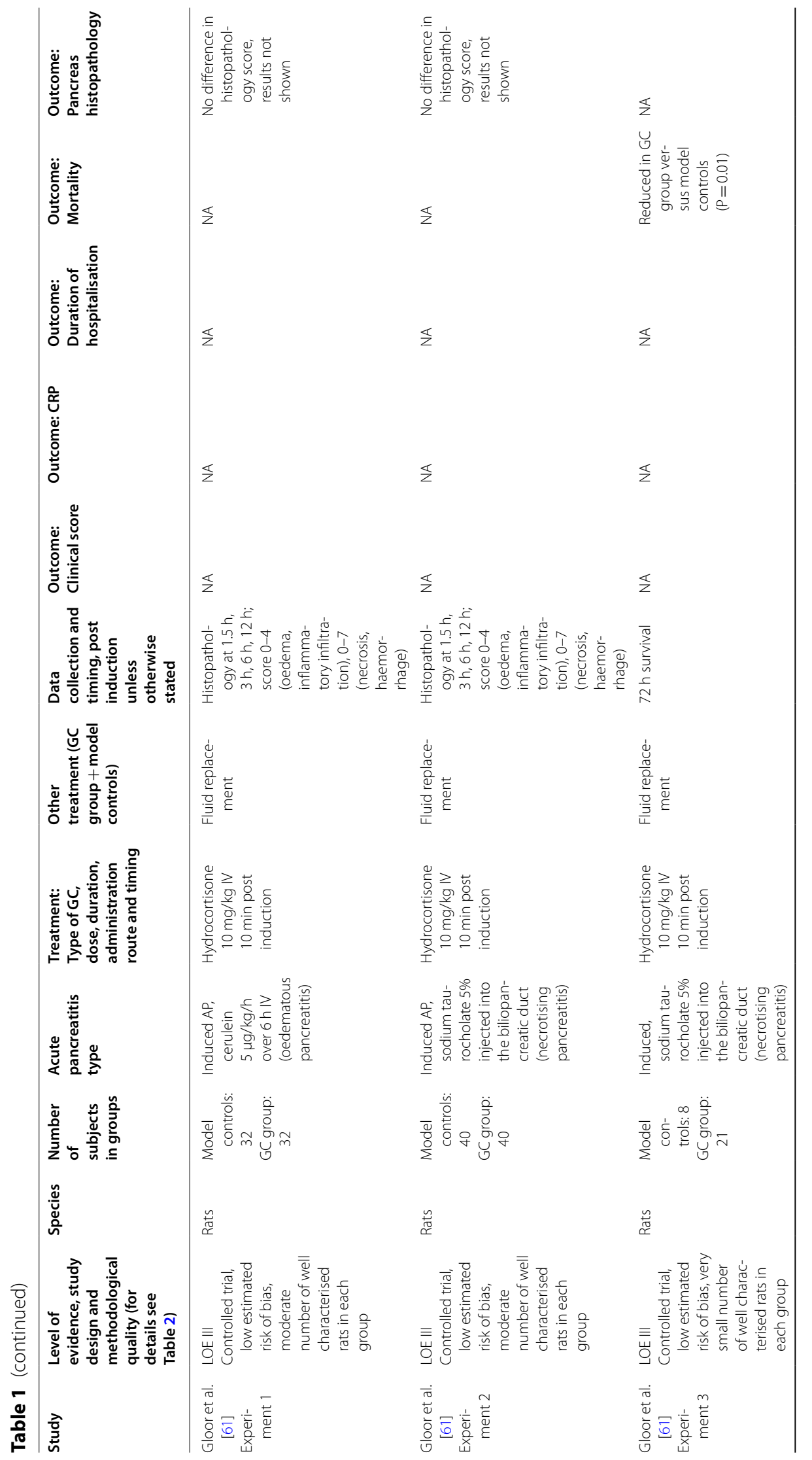




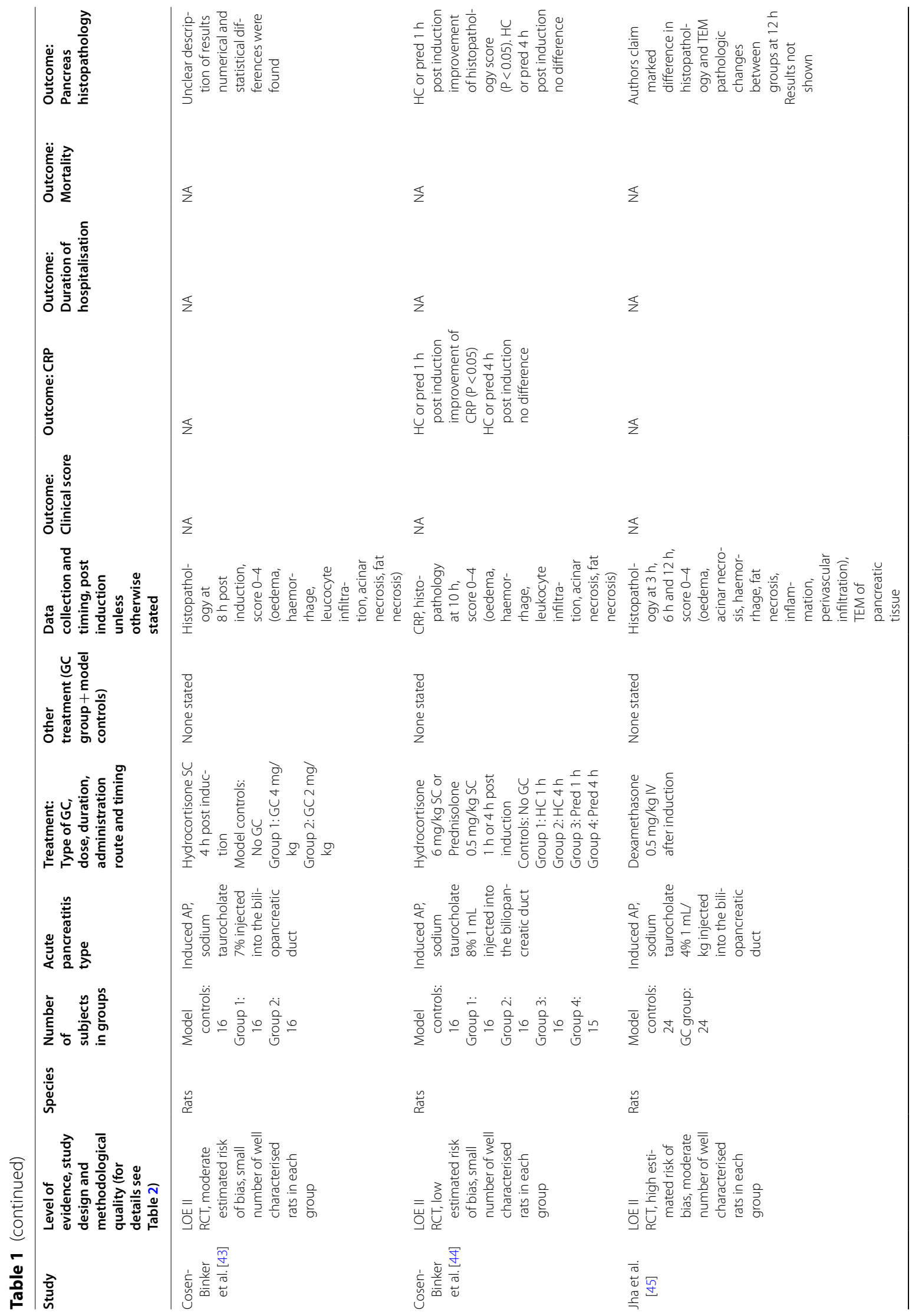




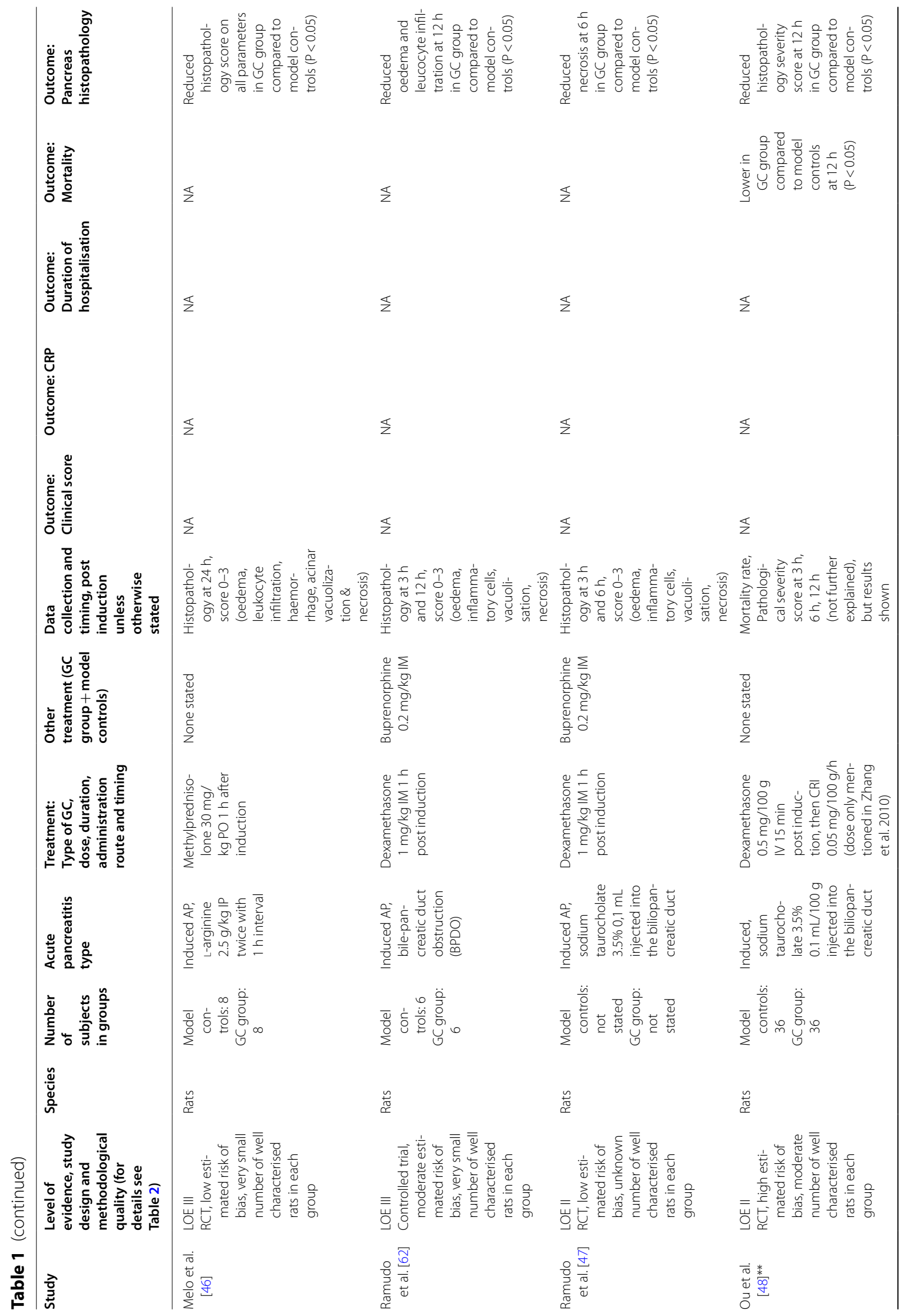




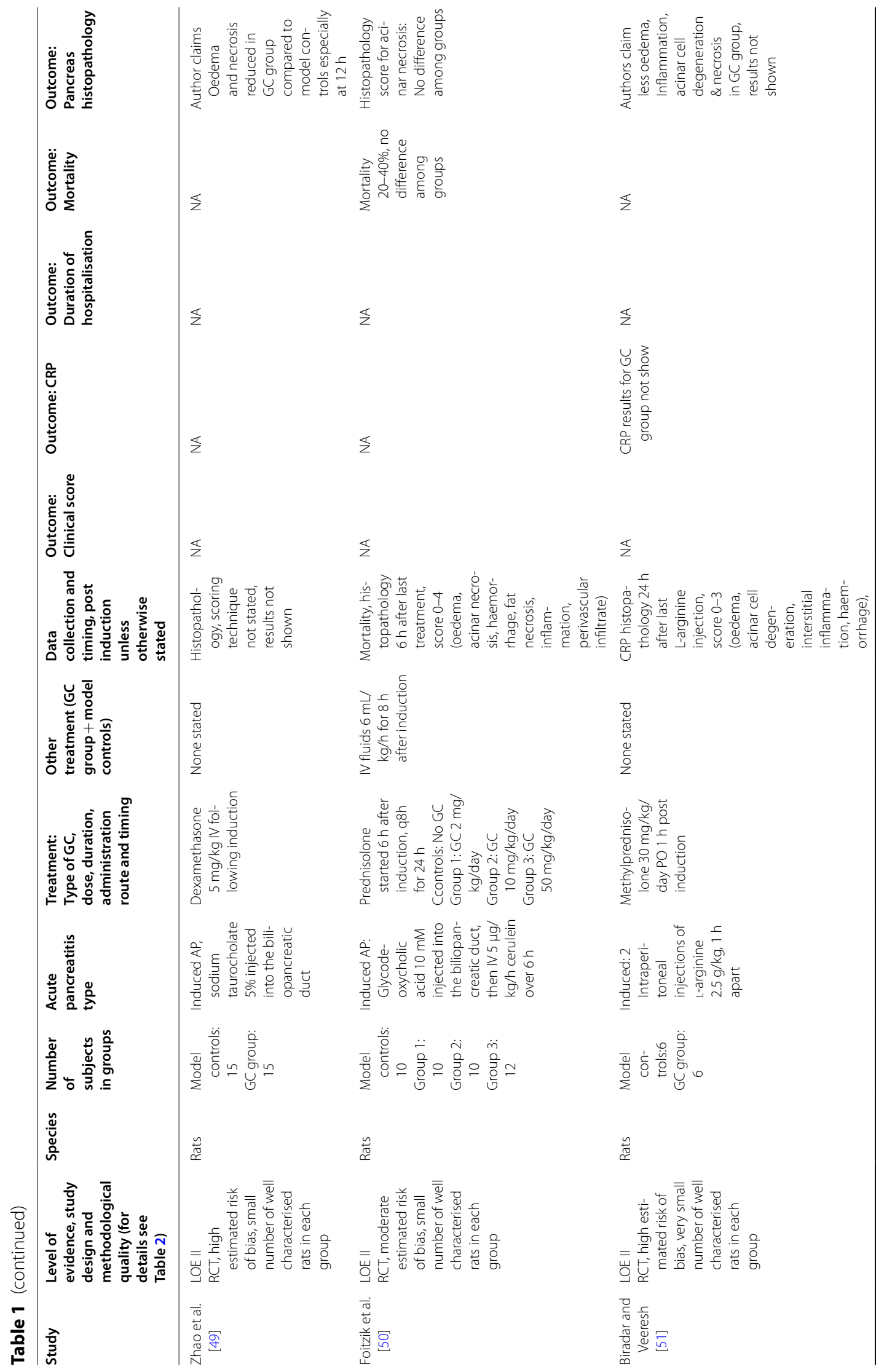




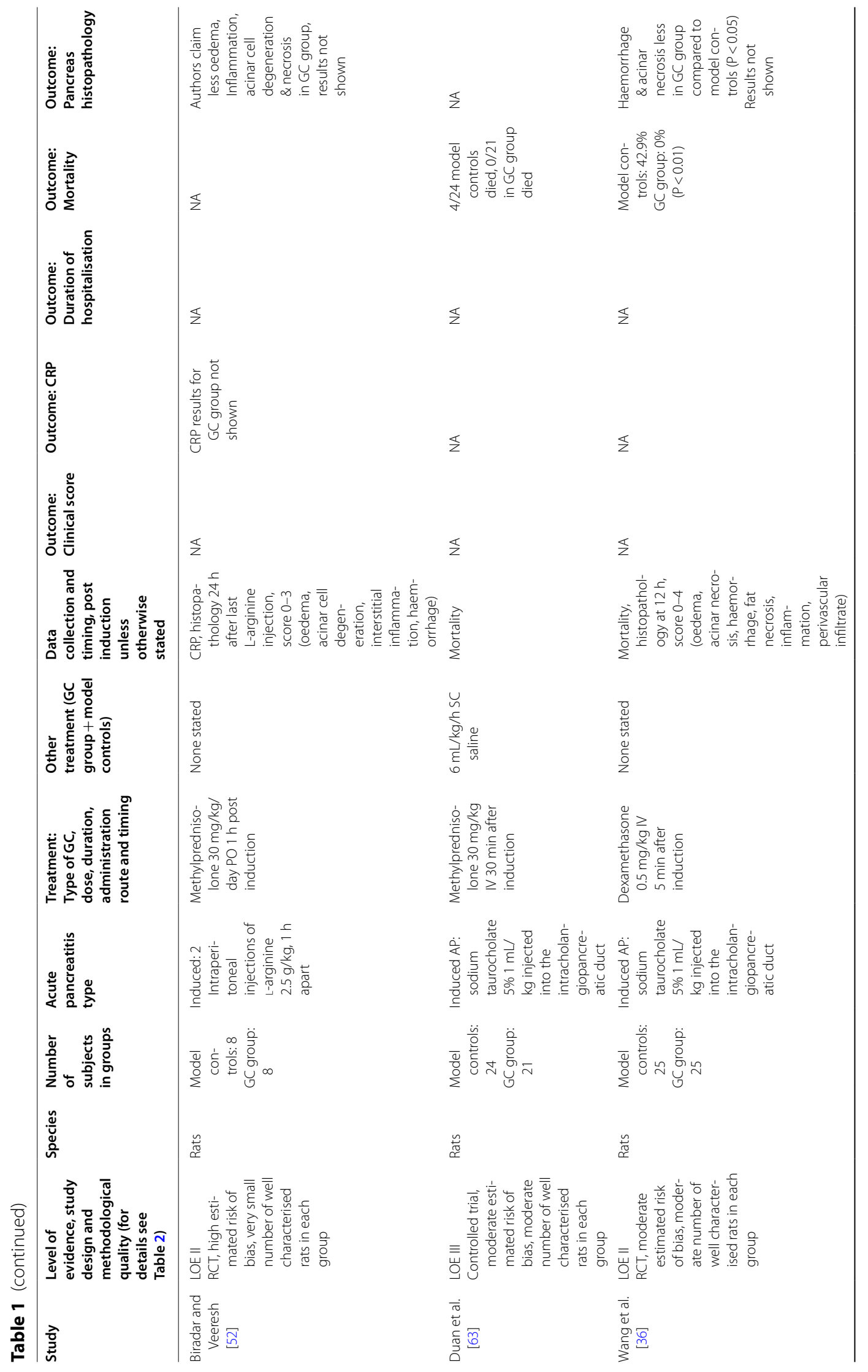




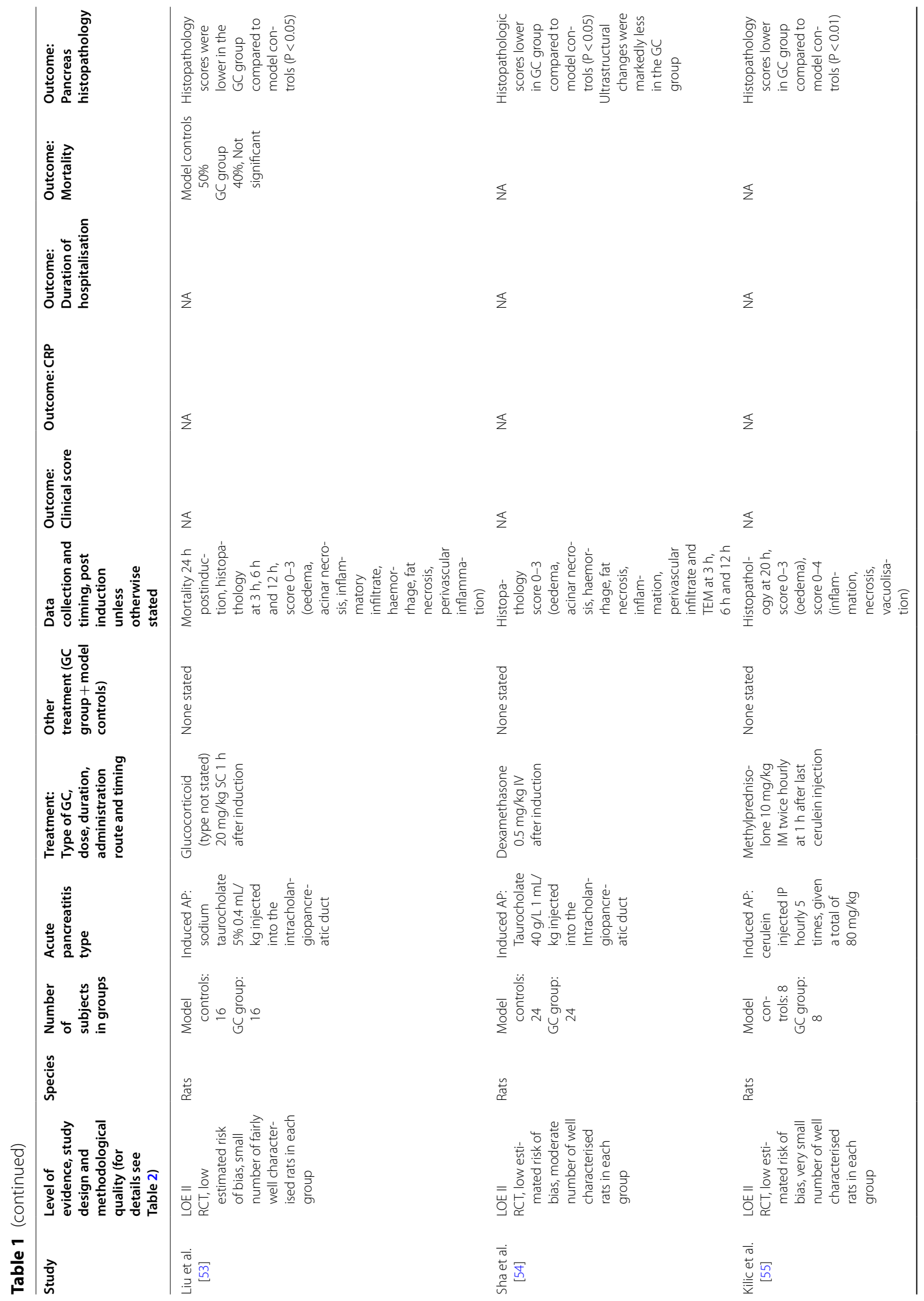




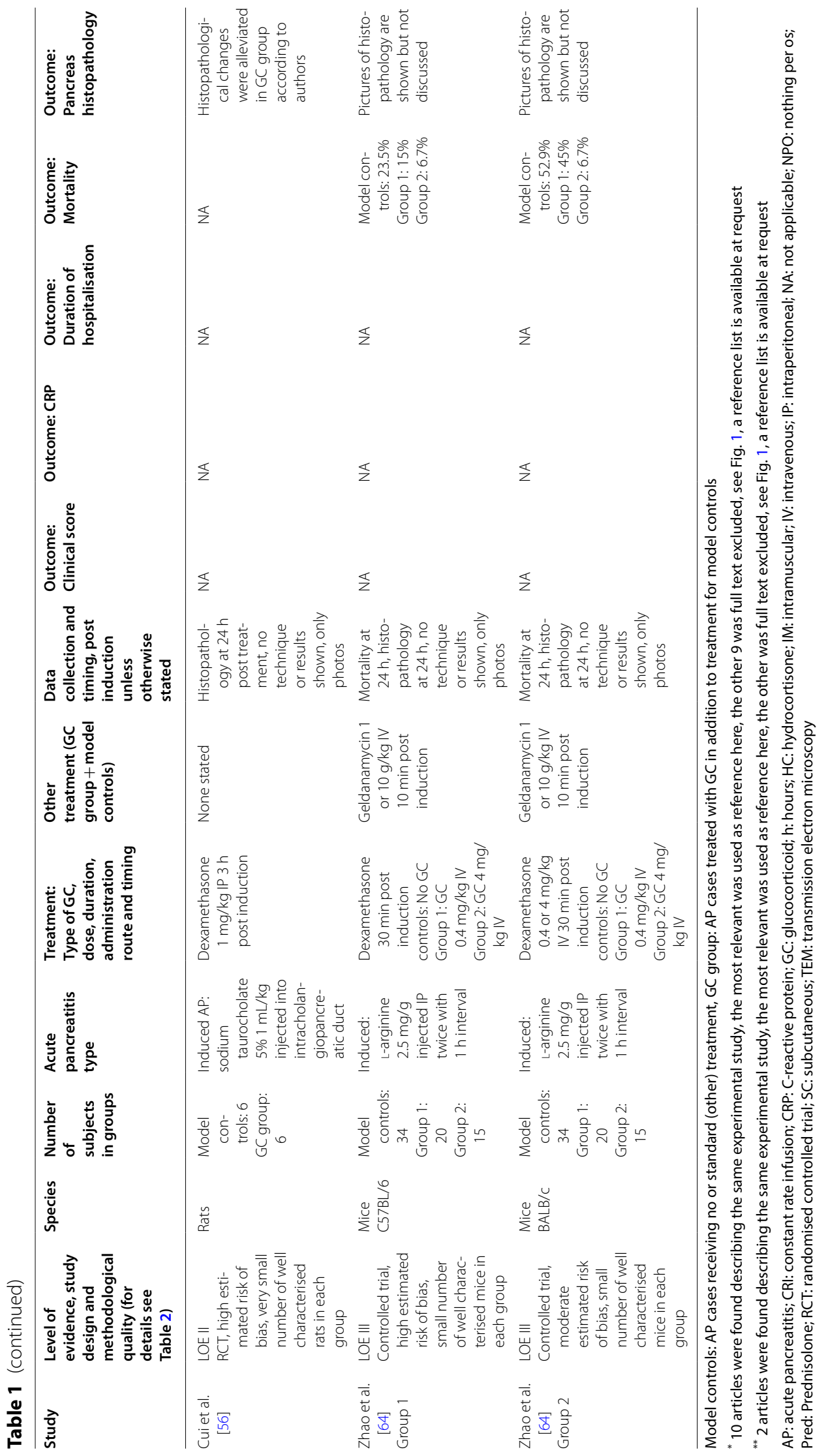




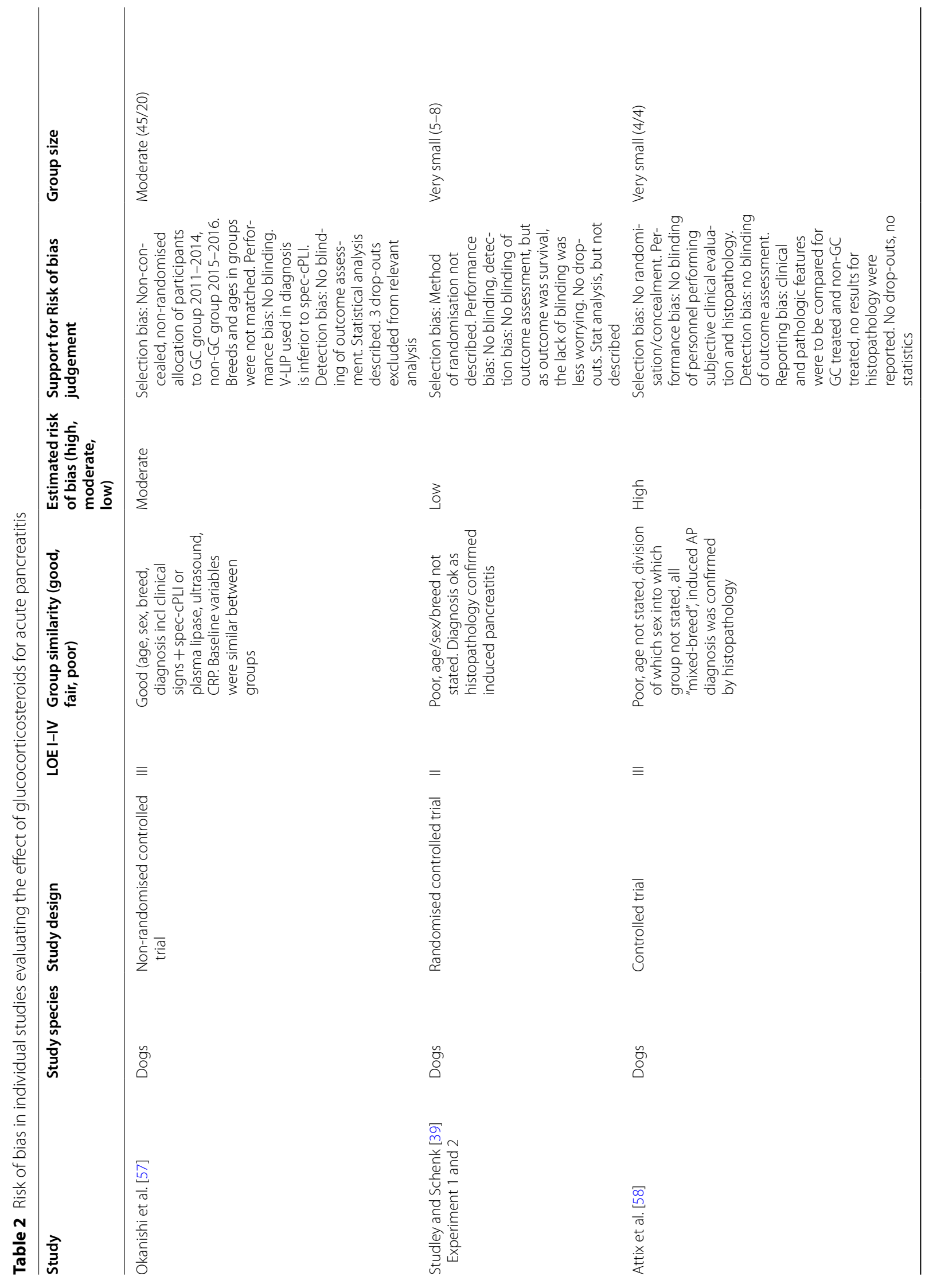




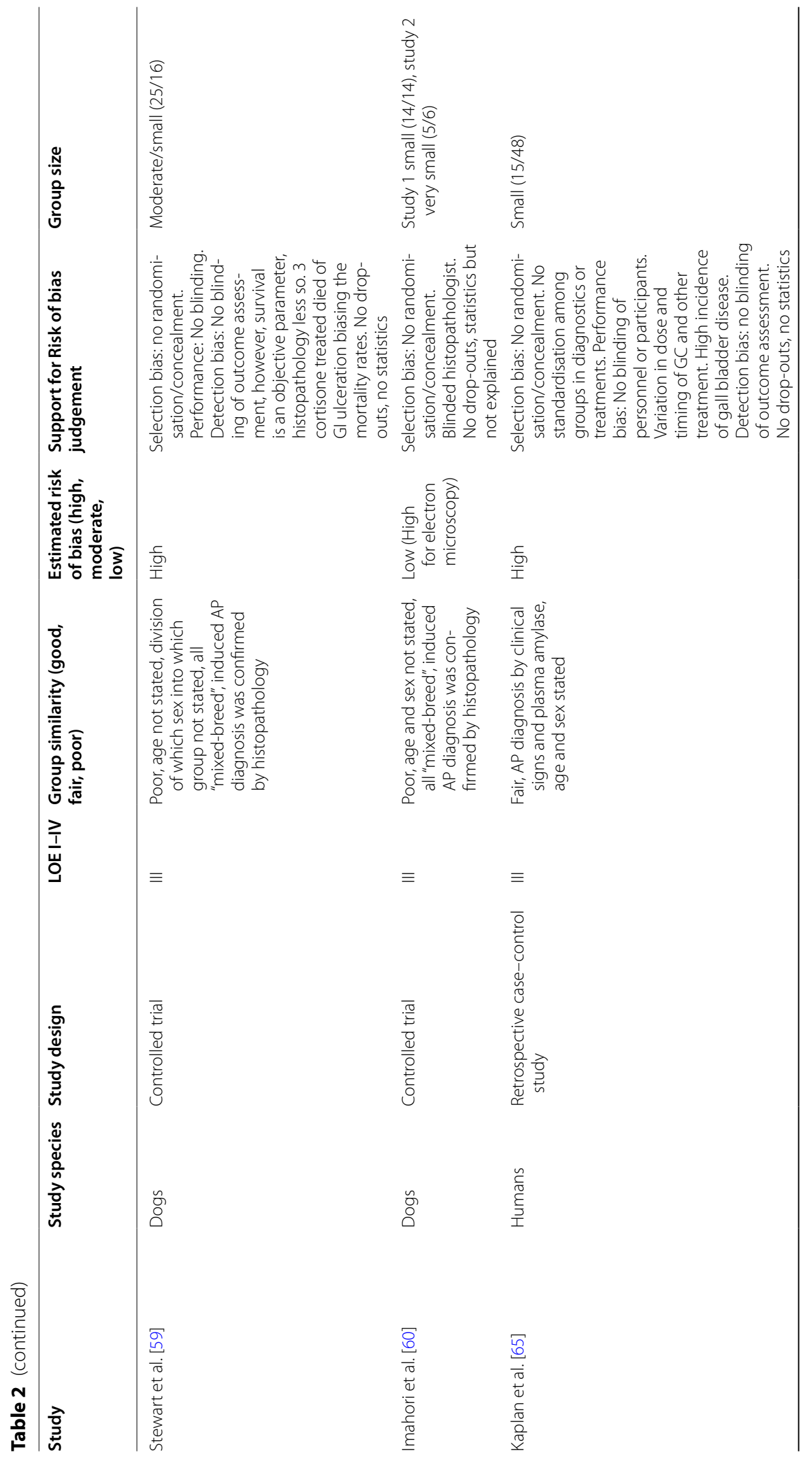




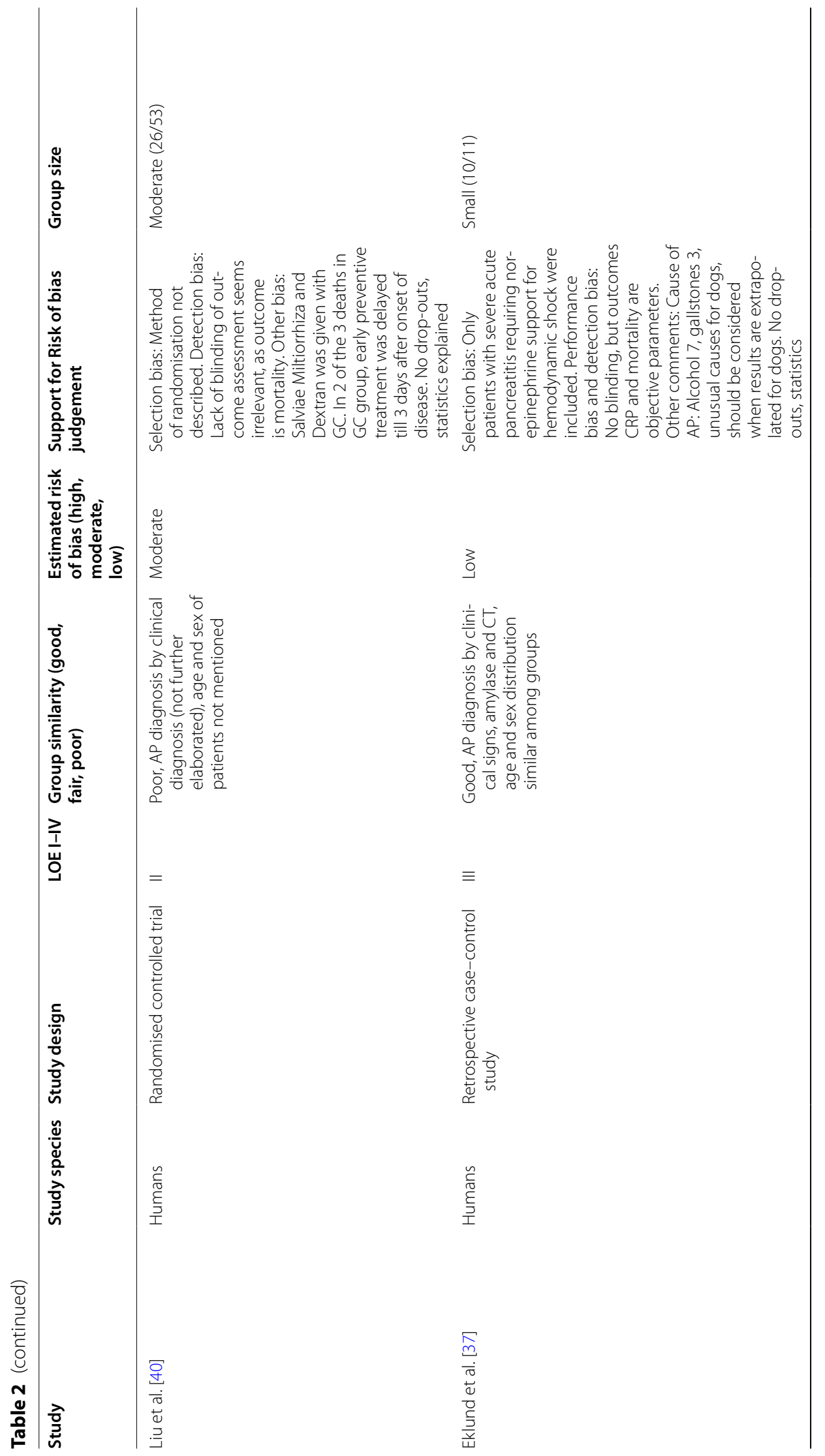




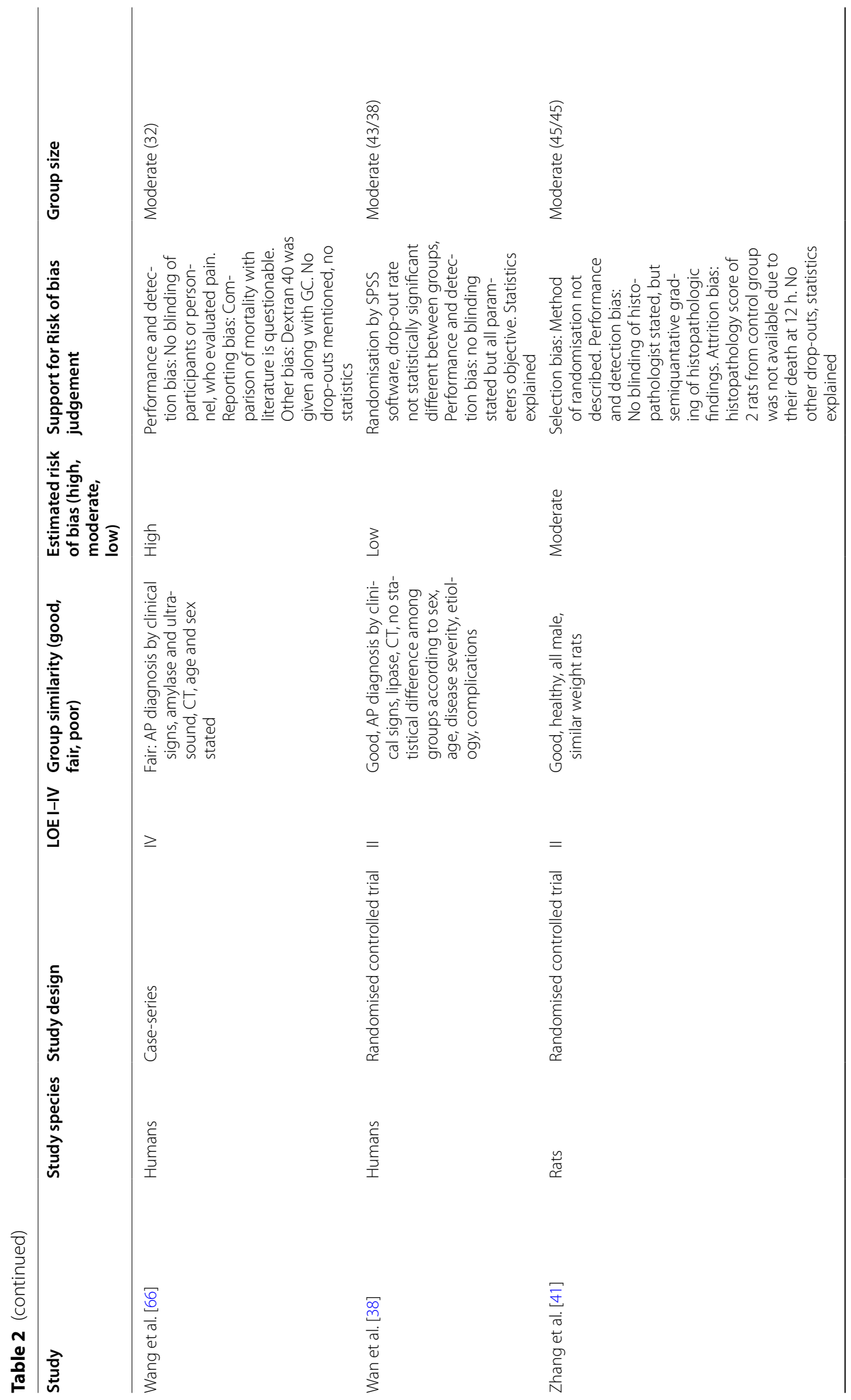




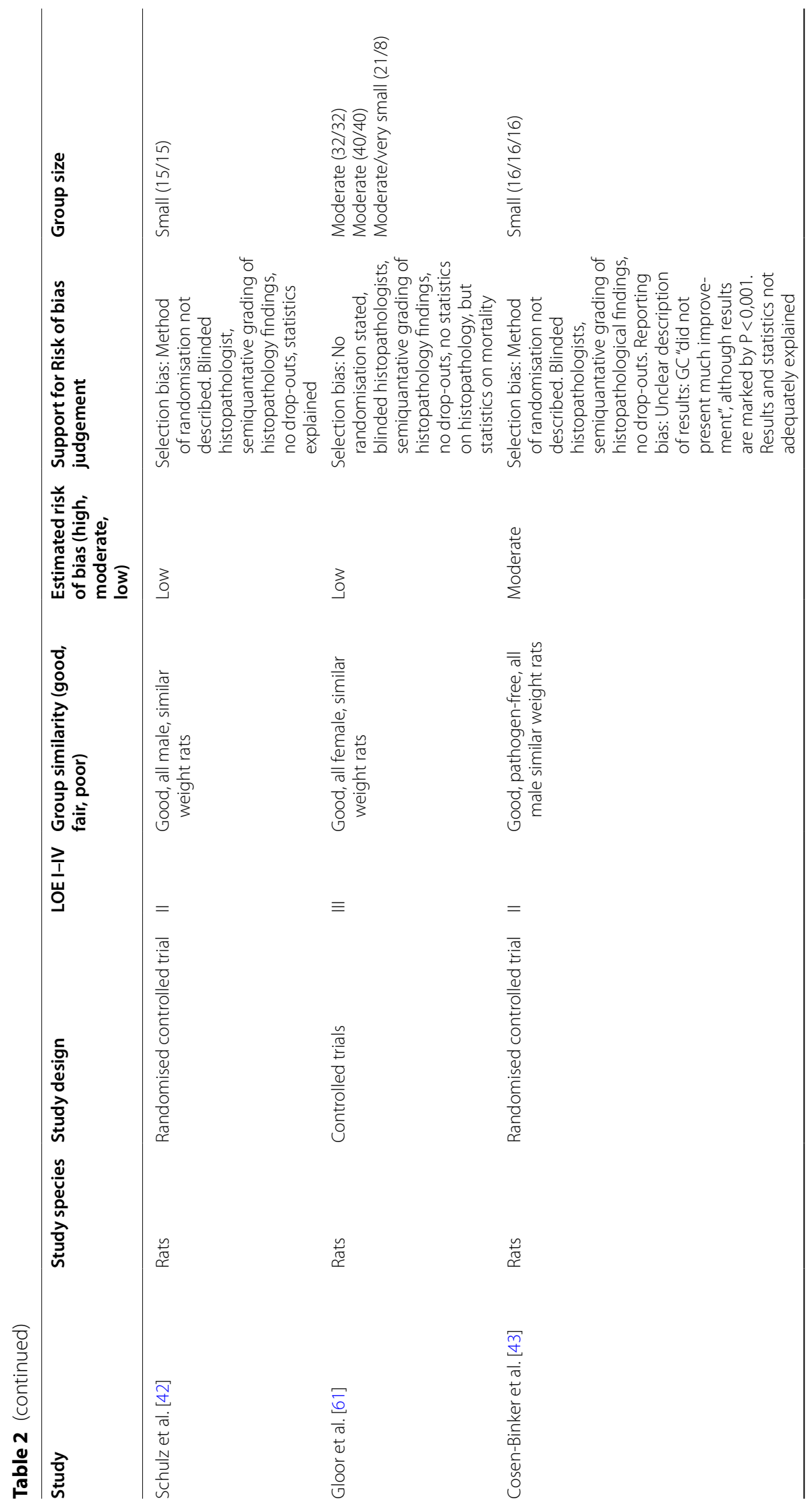




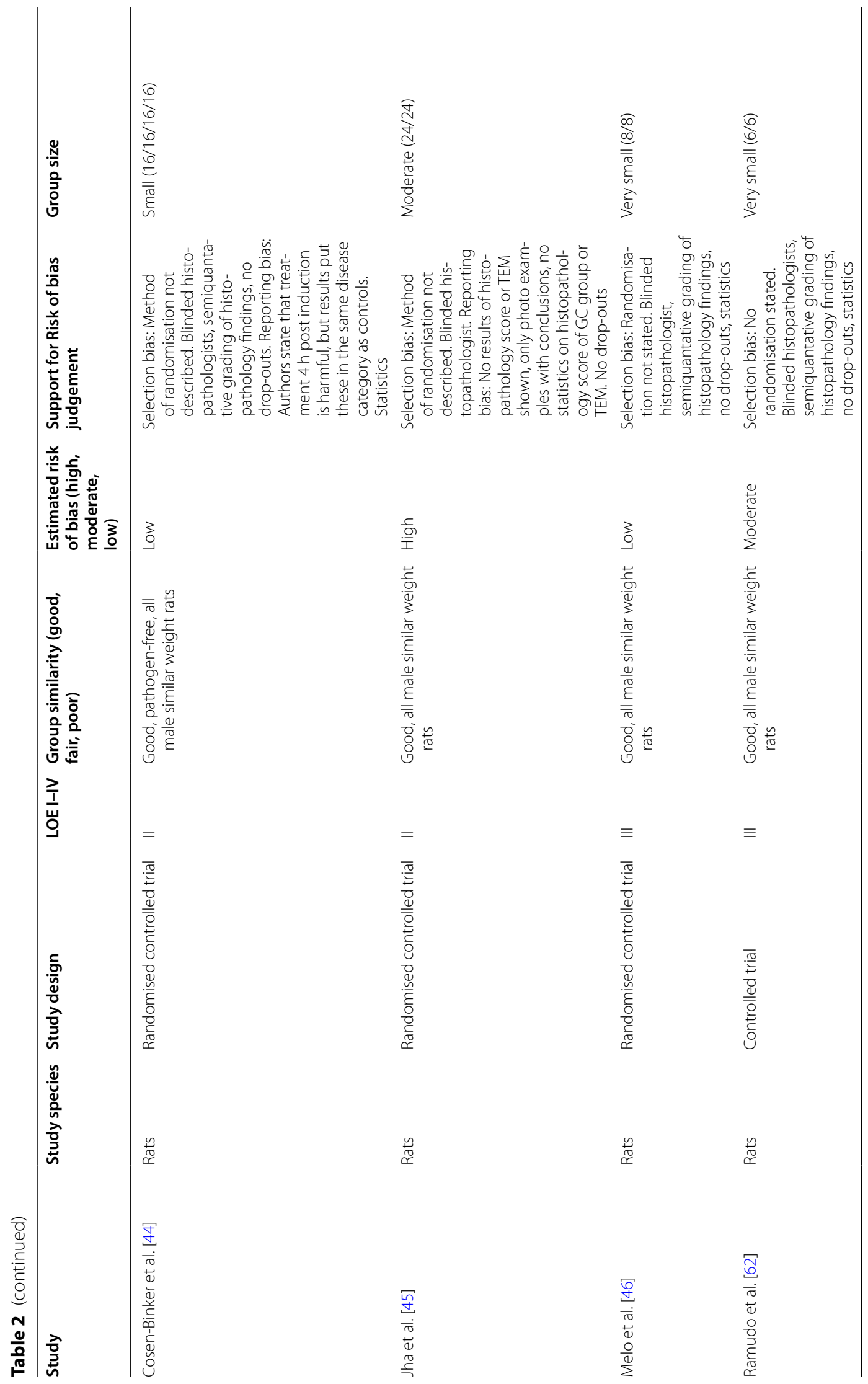




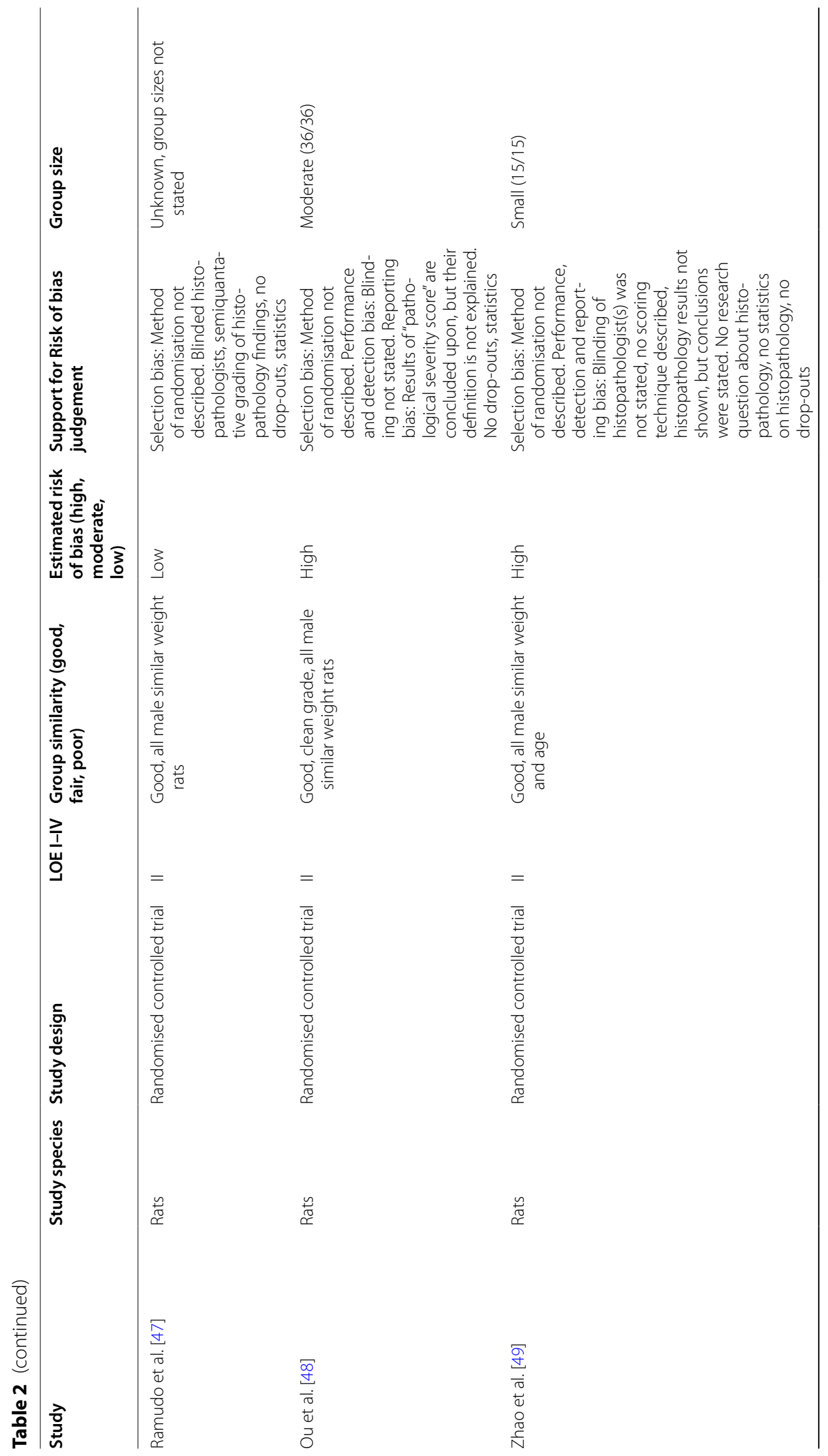




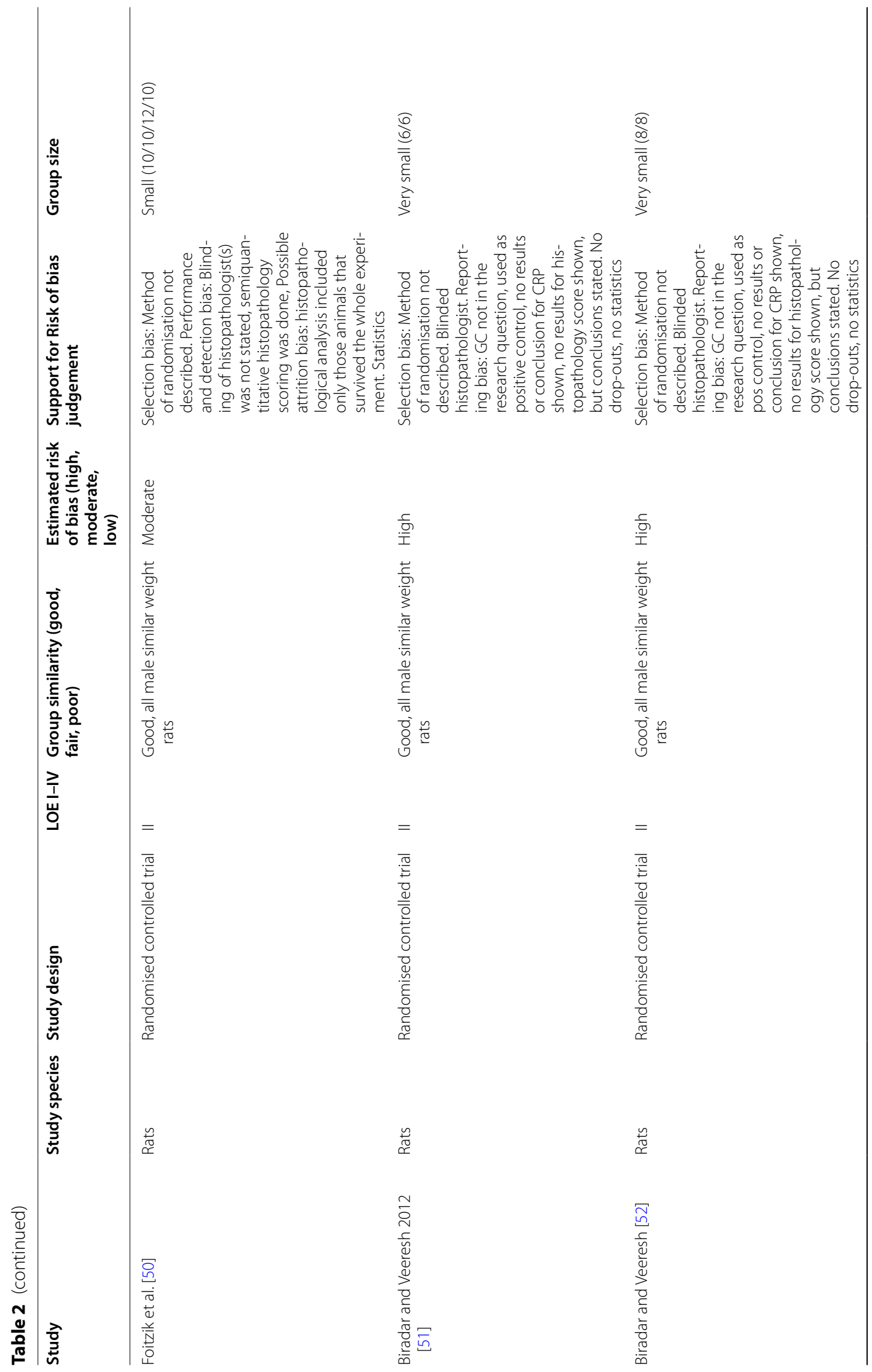




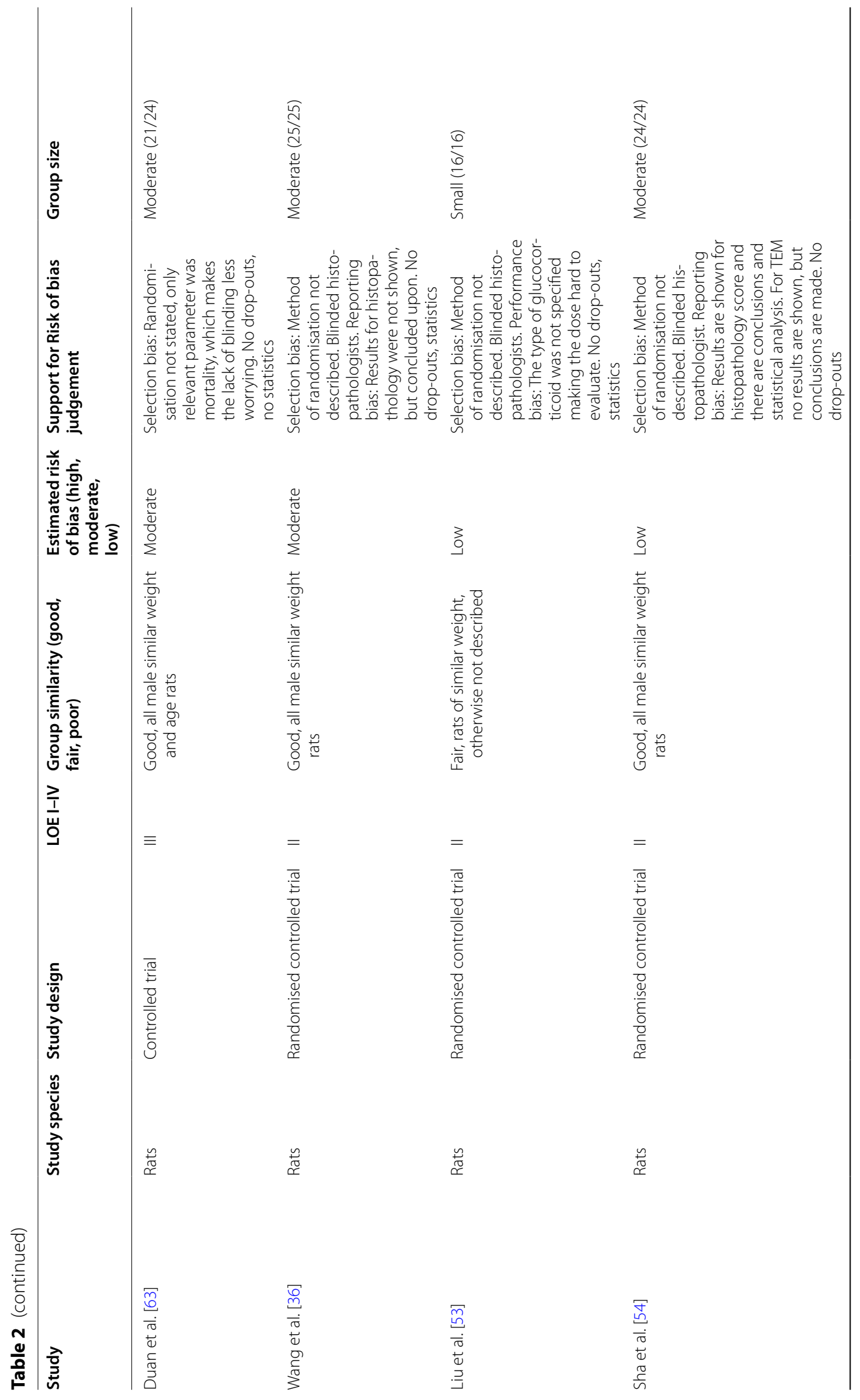




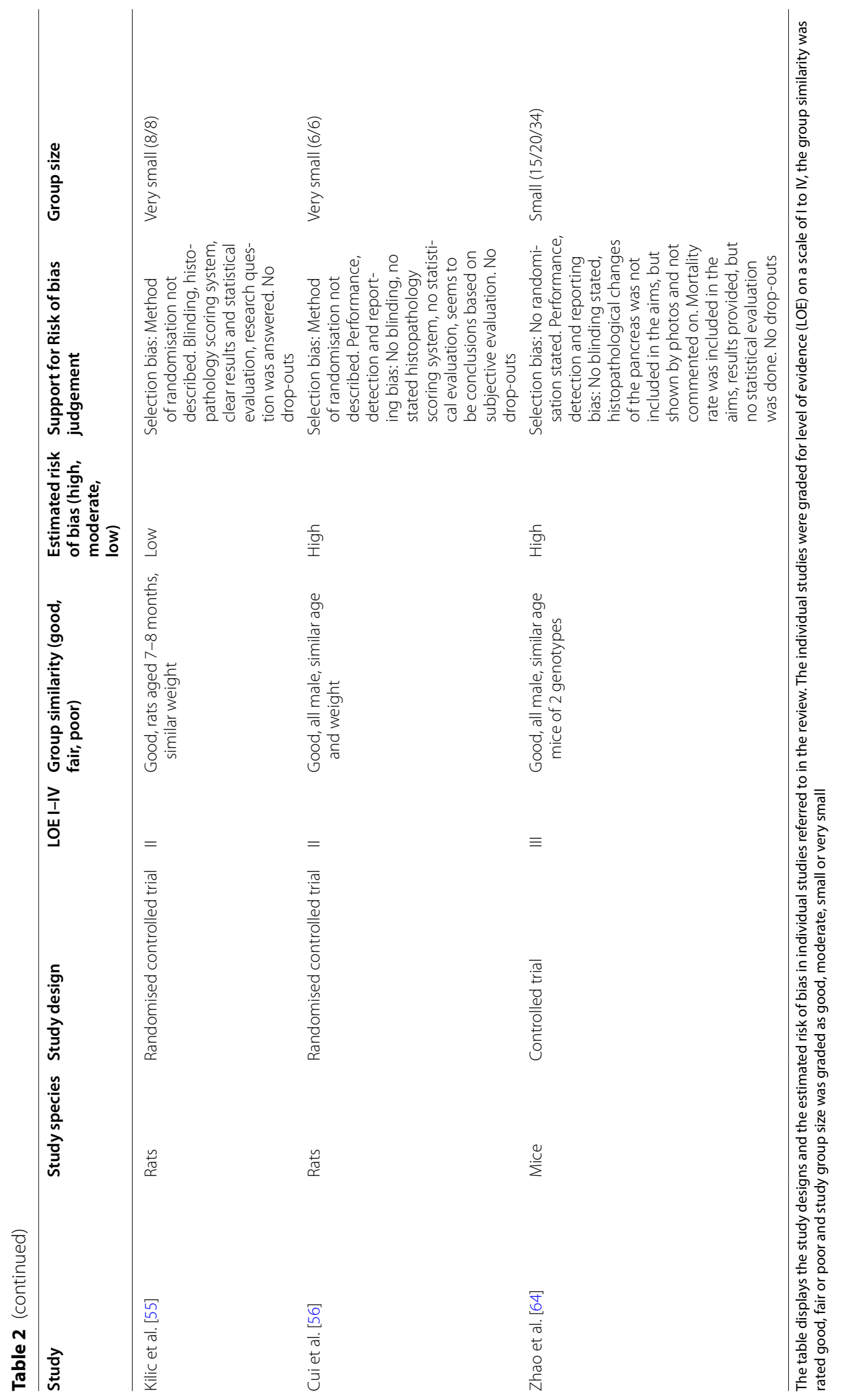


survival [36, 48, 61], and 3 studies found no significant difference in survival between corticosteroid-treated and model controls $[42,50,53]$. A comparison of the studies highlighted certain features. Five out of 6 studies used the same mode of AP induction, and all studies reported high mortality rates of non-treated rodents (20-86\%). However, the type, dose and timing of the corticosteroid treatment varied greatly. For the studies reporting no difference in mortality, a dose of 2, 10 and $50 \mathrm{mg} / \mathrm{kg}$ prednisolone [50], $25 \mathrm{mg} / \mathrm{kg}$ methylprednisolone [42] or $20 \mathrm{mg} /$ $\mathrm{kg}$ glucocorticoid of unknown type [53] were used, and they were all administered $1 \mathrm{~h}$ or later following induction. For studies reporting significantly improved survival, rodents were treated with $10 \mathrm{mg} / \mathrm{kg}$ hydrocortisone [61], $5 \mathrm{mg} / \mathrm{kg}$ dexamethasone [48] or $0.5 \mathrm{mg} / \mathrm{kg}$ dexamethasone [36] within $15 \mathrm{~min}$ after induction.

Seven studies reported mortality results that were not statistically evaluated [37, 41, 59, 63-66]. Only the experimentally induced AP studies performed histopathological analyses of the pancreas (Table 1). Thirteen studies presented statistically evaluated results. Three of these did not report blinding of the histopathologists [41, $48,50]$. Eleven studies reported significantly less severe histopathological changes in the pancreas of corticosteroid-treated animal groups than in the noncorticosteroid-treated groups. Of these, 7 studies concluded that the pancreas histopathological score was significantly lower in the corticosteroid group than in the model control group. The results are generally based on similar scoring systems for oedema, acinar necrosis, inflammatory infiltrate, and haemorrhage and certain including rating of fat necrosis and perivascular inflammation as well [41, $44,46,53-55]$. One of the studies did not define their grading system [48]. In 4 studies, the following histopathological changes were significantly milder than those in the control groups; acinar necrosis [60], oedema and leucocyte infiltration at $12 \mathrm{~h}$ [62]; necrosis at $6 \mathrm{~h}$ [47]; and haemorrhage and necrosis at $12 \mathrm{~h} \mathrm{[36].} \mathrm{Two} \mathrm{studies}$ found no significant difference between the histopathological findings in rodents treated with corticosteroids and untreated rodents $[42,50]$.

Three studies presented no histopathological results or unclear findings $[43,58,64]$. Seven studies did not present statistical evaluations of their results $[45,49,51,52$, $56,59,61]$. Furthermore, in 2 studies that presented electron microscopy results for the pancreas $[45,60]$, statistical evaluations were not performed.

\section{Risk of bias across studies}

In the assessment of risk of bias that could influence the cumulative evidence, Performance and detection bias frequently contributed to a classification of moderate to high risk of bias (Table 2). Several studies reported no blinding of outcome assessment of subjective parameters, such as clinical score and histopathology [41, 48-50, $56,58,59,65,66]$.

Reporting bias had the potential to severely influence the cumulative evidence, especially in cases where histopathological examinations were performed without scoring guidelines, results were not reported or statistical evaluations of the results were not performed but conclusions were made nonetheless $[36,45,48,49,51,52,56]$.

\section{Discussion}

\section{Summary of evidence}

The aim of the present review was to evaluate whether dogs with acute/acute-on-chronic pancreatitis that received standard treatment could benefit from additional treatment with corticosteroids to improve disease outcome as indicated by the clinical score, circulating CRP levels, hospitalisation duration, mortality rate and pancreas histopathologic evaluation.

For the clinical score, the overall evidence was insufficient. There were no RCTs with a low risk of bias or a moderate risk of bias and moderate study size to establish recommendations regarding the use of steroids to reduce clinical scores.

Regarding the circulating CRP levels, one RCT with a low estimated risk of bias [44] concluded that treatment within $1 \mathrm{~h}$ was significantly beneficial while treatment within $4 \mathrm{~h}$ was not. Hence, fair evidence was found that corticosteroid treatment can reduce CRP levels if given early in the disease process. Furthermore, a canine nonrandomised controlled trial and a human case control study found significant reductions in circulating CRP levels in patients treated with corticosteroids compared to the model controls $[37,57]$.

Fair evidence was found that corticosteroid treatment can shorten hospitalisation length. This evidence was based on 1 canine and 1 human study in which a significantly shortened duration of hospitalisation was reported in the corticosteroid-treated groups compared to the model controls $[38,57]$. The latter was a RCT with low estimated risk of bias.

The overall evidence for corticosteroid treatment reducing mortality in AP is conflicting. Seven studies provided results of significantly improved survival of corticosteroid-treated patients compared to model controls. Of these, 3 studies were RCTs of low risk of bias or moderate risk of bias along with moderate study size, thus providing good evidence for improved survival with corticosteroid treatment of AP [36, 39, 40]. However, 4 studies found no significant difference in survival and all were RCTs presented a low estimated risk of bias or moderate risk of bias along with moderate study size [38, 41, $42,53]$, thus providing good evidence that corticosteroid 
treatment is of no significant benefit for survival. Notably, in rodent studies with improved survival [36, 48, 61], corticosteroids were administered early in the experimentally induced disease process (within $15 \mathrm{~min}$ ), whereas in most of the studies without improved survival $[42,50,53]$, corticosteroids were given later in the disease process ( $1 \mathrm{~h}$ or later).

The overall evidence that corticosteroid treatment results in less severe histopathological changes in experimentally induced pancreatitis is good. Eight RCTs with low estimated risk of bias or moderate risk of bias that also had a moderate study size found significantly less severe histopathological changes in the pancreas in the corticosteroid-treated animal groups than in the model controls [36, 41, 44, 46, 47, 53-55]. One RCT with a low estimated risk of bias provided fair evidence that corticosteroid treatment results in no significant improvement of histopathology [42].

\section{Limitations}

The most obvious limitation of this review is the limited number of studies performed in dogs. Therefore, the results are useful for providing general conclusions on acute pancreatitis but insufficient for generating robust conclusions on canine acute pancreatitis. Among the 5 canine studies included, only the work of Studley and Schenk [39] was a RCT with a low estimated risk of bias; thus, it was the only canine study with the power to grade evidence for the general results in this review. Nevertheless, 3 of the 4 other canine studies were consistent with the positive effect of corticosteroids reported by Studley and Schenk [39]. A controlled clinical trial of moderate group size was recently reported in Okanishi et al. [57], and they found a significant benefit of prednisolone treatment with regard to clinical score, CRP level, hospitalisation duration and survival in dogs. Stewart et al. [59] and Imahori et al. [60] both found a positive corticosteroid effect on survival and pancreas histopathology in dogs with experimentally induced pancreatitis. A striking difference between these studies and a previous canine study, in which corticosteroids did not confer a benefit [58], was the timing of corticosteroid treatment start. The above 4 studies started treatment early in the disease process (at diagnosis, or no later than $12 \mathrm{~h}$ after experimental induction) and the study by Attix et al. started treatment $24 \mathrm{~h}$ after induction [58].

Similarly, early treatment was supported in the included rodent studies. No positive effect of corticosteroid treatment on mortality was observed in studies that started treatment $1 \mathrm{~h}$ or later after induction, while a positive effect was frequently observed in studies that started treatment early (within $15 \mathrm{~min}$ ). Cosen-Binker et al. [44] concluded that hydrocortisone or prednisolone administered $1 \mathrm{~h}$ after induction resulted in slight but significant improvement of histopathology score and CRP levels while the same treatments administered $3 \mathrm{~h}$ later did not. Overall, the findings indicate that the early start of corticosteroid treatment might be important for a positive effect.

Cosen-Binker et al. [44] indicated that hydrocortisone and prednisolone seemed to have the same effect, and Studley and Schenk [39] did not observe differences in the positive effect of low or high doses of hydrocortisone. In the included studies, a wide range of types and doses of corticosteroids were used, and identifying associations between the type or dose of corticosteroids and the effect was difficult. Any association found in this multispecies review would be of questionable use for specific canine treatment recommendations.

Another limitation of this review was that two of the human studies involved treatments with dextran or Salvia miltiorrhiza along with corticosteroids. Although these treatments are not recommended by international treatment protocols and could bias the results $[40,66]$, one of these studies was included here as evidence for reduced mortality [40].

Another limitation of the current review is the use of studies concerning experimentally induced acute pancreatitis because this disease does not necessarily mimic spontaneous disease. In particular, the severity of the induced disease, which is typically described as severe acute pancreatitis with tissue necrosis and mortality rates up to $86 \%$ [61], is not directly comparable with the veterinary clinical setting, where the severity of acute pancreatitis varies from subclinical to very severe.

Two meta-analyses were found during the literature search, one on humans and one on rodents; however, they were excluded and only relevant individual studies were retained. The human meta-analysis included 5 studies, but only one was included in this review [38] because the others were only published in Chinese and hence excluded based on the English language criterion [67]. Consistent with the current review, the human meta-analysis concluded that corticosteroid therapy may improve outcomes in patients with severe acute pancreatitis. From the meta-analysis on rodents, 6 studies were included [36, 41, 47, 48, 61, 62], the text for one study could not be retrieved, and the remaining studies included pretreatment with steroids and were thus excluded [68]. This meta-analysis also concluded that corticosteroids have beneficial effects on rodent animal models of severe acute pancreatitis.

None of the included studies presented sample size calculations; thus, the possibility of studies being underpowered cannot be ignored. Drop-outs were often poorly described or not described in the included studies. 
However, drop-outs were considered to have a minor influence on the results, especially for the experimental studies, where animals typically were closely followed for a short period of time (hours or a few days). Similarities at baseline were reasonable, and only 7 of the 31 studies rated poor or fair. Older human and canine studies presented less than adequate descriptions. The level of evidence was also reasonable among the studies, with just 1 study graded LOE IV.

Acute pancreatitis is known to be a very painful condition. As most of the experimental studies had been evaluated by an ethical committee, one might wonder why only 2 of the experimental studies used analgesics in addition to corticosteroids, which meant that all animals in the control groups were not provided pain relief. Pain could very possibly influence the disease outcome and results.

In this review, 5 disease outcome parameters were evaluated. Clinical scores were reported in 4 studies; however, they were all differently defined, thereby limiting any conclusions on this parameter.

CRP is a major acute phase protein in several species, including mice, humans and dogs. It is one of the most sensitive markers of inflammation in dogs and used to confirm the presence of underlying inflammation and monitor the response to therapy $[69,70]$. Furthermore, CRP testing is widely used in clinical practice. Therefore, CRP was included as an outcome parameter. In earlier human guidelines on acute pancreatitis, a CRP level $>150 \mathrm{mg} / \mathrm{L}$ was indicative of severe pancreatitis [71]. However, later studies found that CRP measured at the first visit is not a reliable prognostic marker for human acute pancreatitis because CRP peak levels are reached only after $48-72 \mathrm{~h}$ [72]. Kuzi et al. [4] found that CRP correlated significantly with CPLI and the canine acute pancreatitis severity score (CAPS), and CRP levels in survivors and nonsurvivors were similar on day 0 and day 1 . Nevertheless, CRP levels measured 48-72 h following initial presentation might correlate with acute pancreatitis outcome. Holm et al. found that CRP was significantly elevated in dogs with spontaneous acute pancreatitis compared with healthy controls, and it decreased significantly from day 1 and 3 to day 5 in association with clinical improvement [73]. Sato et al. [74] found significantly lower CRP levels on days 3 and 4 in survivors compared with nonsurvivors among dogs with acute pancreatitis. In the present review, one human study [37], one canine study [57] and one rodent study [44] followed CRP over time. In the human study, there was a significant difference in CRP levels between HCtreated and nontreated patients at $48 \mathrm{~h}$ [37], and Okanishi et al. [57] found a significant difference between prednisolone-treated and nontreated dogs with acute pancreatitis on day 3 . However, whether these improvements in CRP levels reflect a true reduction in inflammation and disease severity or a direct suppression of IL-6, which is the main inducer of hepatic CRP production, is not known. Many other parameters have been reported to be improved by corticosteroid treatment and could have been evaluated, including ascites [50], acute respiratory distress syndrome $[38,40]$, time to vasopressor cessation [37], arachidonic acid breakdown products [61], amylase, lipase, TNF $\alpha$, and IL-6 levels [46, 51], and morphological changes of the lungs [54].

The reason why corticosteroids may positively affect the development of acute pancreatitis could be related to findings in human studies of relative adrenal insufficiency (RAI), which is also called critical illness-related corticosteroid insufficiency (CIRCI). Clinical studies have found that human patients suffering from severe acute pancreatitis have low corticosteroid baseline levels and/or poor response to ACTH stimulation. RAI/CIRCI in severe acute pancreatitis patients has been associated with higher clinical severity scores, pancreatic necrosis, need for surgical intervention and higher mortality [75, 76]. These findings, along with the abovementioned human meta-analysis by Dong et al. [67], have led to the cautious recommendation of corticosteroid therapy for severe acute pancreatitis in humans by some authors [77]. However, to the authors knowledge, this recommendation has not yet been implemented in any official treatment guidelines of human acute pancreatitis. Additionally, whether RAI is present in dogs with acute pancreatitis and whether it relates to survival is still controversial $[78,79]$.

As mentioned in the introduction, it is believed that CAP becomes life-threatening with the development of disseminated intravascular coagulation and multiple organ failure [2], and it could therefore be speculated that the use of corticosteroids in the treatment of CAP could be of particular benefit in severe cases at risk of developing these complications. Recent evidence has shown that corticosteroid treatment is beneficial for COVID-19 patients needing oxygen therapy or ventilation but not for patients managing without such therapy [80]. A common feature of human patients with severe acute pancreatitis and COVID-19 is the excessive formation of neutrophil extracellular traps (NETs) by activated neutrophils, leading to thrombosis and multiorgan damage [81, 82]. NETosis has been recognised in several species, including humans, mice and dogs [83]. Human patients with acute pancreatitis have increased levels of circulating NETs compared with healthy controls [84], and in mice, NETs can directly induce trypsin formation; moreover, mitigation of NET formation reduces pancreatic inflammation and lung damage [85]. Corticosteroids have been shown to downregulate NETs in the lungs of 
an experimental equine model of asthma [86], and in humans with chronic inflammatory lung disease, NETs were decreased in patients who frequently used corticosteroid inhalers [87]. The important role of neutrophils in disease progression is further supported by the recent approval in Japan of the drug fuzapladib sodium hydrate (BRENDA $^{\mathrm{TM}} \mathrm{Z}$, Ishihara Sangyo Kaisha Ltd. Osaka, Japan) for improving clinical signs in the acute phase of pancreatitis in dogs [88]. Fuzapladip sodium hydrate prevents the translocation of neutrophils from blood vessels to the extravascular tissue. It is not as broad-acting as corticosteroids and may have fewer side effects; however, until it is approved for global use, early short-term corticosteroid treatment could be considered for mitigating NET formation.

In addition to the anti-inflammatory effect, corticosteroids may also act as procoagulants, which could be an unwarranted effect in patients with acute pancreatitis. Therefore, based on current data, specific CAP subgroups may respond differently to corticosteroid treatment. Whether subgroups exist and how they should possibly influence recommendations on the dosage, treatment initiation and duration needs further investigation. When considering corticosteroid dosage and treatment duration, practitioners should keep in mind that studies have found evidence of corticosteroids causing or increasing the risk of acute pancreatitis [21, 22].

\section{Conclusions}

The current review of 31 studies evaluating corticosteroid treatment of acute pancreatitis provides fair evidence indicating the beneficial effect of corticosteroids on the duration of hospitalisation and circulating CRP levels. With respect to mortality rate and histopathological severity, a clear conclusion is more difficult to reach. Good evidence has been provided for a decreased mortality rate with corticosteroid treatment, while at the same time, good evidence has been provided of a lack of significant effect. Good evidence has been provided for a lower histopathological score of pancreatic changes with corticosteroid treatment, while fair evidence has also been provided for a lack of difference.

These findings lead to the cautious conclusion that additional treatment with corticosteroids in the acute/ acute-on-chronic phase of canine pancreatitis might have a positive influence on disease outcome.

Because the evidence in this review was gathered for several species and included experimentally induced pancreatitis, specific treatment recommendations cannot be provided regarding the type, timing and dosage for dogs with CAP in the clinical setting.

In conclusion, although corticosteroid treatment of CAP may be beneficial, this review recommends larger randomised controlled studies in dogs with spontaneously occurring acute/acute-on-chronic pancreatitis to further elucidate the effect of corticosteroid treatment, determine the type, dose and length of corticosteroid treatment and identify possible CAP patient subgroups that could possibly benefit.

\section{Supplementary Information}

The online version contains supplementary material available at https://doi. org/10.1186/s13028-021-00592-0.

Additional file 1. Search strategy. A list of search terms used in the database literature search is provided to elucidate the search process. The list is uploaded separately in Microsoft Word format.

\section{Acknowledgements}

Not applicable.

\section{Authors' contributions}

The review was performed by KBN as a thesis for a master's degree in companion animal internal medicine at the University of Copenhagen. CRB supervised the process and substantially contributed to the conception of the review. Both authors have reviewed and discussed the included studies. Both authors read and approved the final manuscript.

\section{Authors' information}

CRB is a professor in Internal Medicine for Companion Animals. Her main research focus is gastrointestinal diseases, obesity, diabetes and metabolism in dogs and cats. She has published several original manuscripts and two systematic reviews prior to this scoping review.

KBN is a practising veterinarian specialising in companion animal internal medicine, and she developed the idea for the current review during her work with canine acute pancreatitis.

\section{Funding}

Not applicable.

Availability of data and materials

All data are included in the article. Information on excluded studies can be obtained from the corresponding author upon reasonable request.

\section{Declarations}

Ethics approval and consent to participate

This study did not require official or institutional ethical approval.

Consent for publication

Not applicable.

\section{Competing interests}

The authors declare that they have no competing interests.

\section{Author details}

${ }^{1}$ Dyrlægehuset Skanderborg, Ladegaardsbakken 16, 8660 Skanderborg, Denmark. ${ }^{2}$ Department of Veterinary Clinical Sciences, Faculty of Health and Medical Sciences, University of Copenhagen, Dyrlægevej 16, 1870 Frederiksberg C, Denmark.

Received: 4 February 2021 Accepted: 2 July 2021

Published online: 13 July 2021 


\section{References}

1. Mansfield C. Acute pancreatitis in dogs: advances in understanding, diagnostics, and treatment. Top Companion Anim Med. 2012;27:123-32.

2. Mansfield C. Pathophysiology of acute pancreatitis: Potential application from experimental models and human medicine to dogs. J Vet Intern Med. 2012;26:875-87

3. Talukdar R, Sareen A, Zhu H, Yuan Z, Dixit A, Cheema H, et al. Release of cathepsin $b$ in cytosol causes cell death in acute pancreatitis. Gastroenterology. 2016;151:747-58.

4. Kuzi S, Mazaki-Tovi M, Suchodolski JS, Rimer D, Lidbury JA, Steiner JM, et al. Protease inhibitors, inflammatory markers, and their association with outcome in dogs with naturally occurring acute pancreatitis. J Vet Intern Med. 2020;34:1801-12.

5. Aupperle-Lellbach H, Torner K, Staudacher M, Stadler C, Tress U, Grassinger JM, et al. Histopathological findings and canine pancreatic lipase immunoreactivity in normal dogs and dogs with inflammatory and neoplastic diseases of the pancreas. J Vet Intern Med. 2020;34:1127-34.

6. Xenoulis PG. Diagnosis of pancreatitis in dogs and cats. J Small Anim Pract. 2015;56:13-26.

7. Watson P. Pancreatitis in dogs and cats: definitions and pathophysiology. J Small Anim Pract. 2015;56:3-12.

8. Watson PJ, Roulois AJ, Scase T, Johnston PE, Thompson H, Herrtage ME. Prevalence and breed distribution of chronic pancreatitis at post-mortem examination in first-opinion dogs. J Small Anim Pract. 2007:48:609-18.

9. Newman SJ, Steiner JM, Woosley K, Williams DA, Barton L. Histologic assessment and grading of the exocrine pancreas in the dog. J Vet Diagn Invest. 2006;18:115-8

10. Bostrom BM, Xenoulis PG, Newman SJ, Pool RR, Fosgate GT, Steiner JM. Chronic pancreatitis in dogs: a retrospective study of clinical, clinicopathological, and histopathological findings in 61 cases. Vet J. 2013;195:73-9.

11. Watson PJ, Archer J, Roulois AJ, Scase TJ, Herrtage ME. Observational study of 14 cases of chronic pancreatitis in dogs. Vet Rec. 2010;167:968-76

12. Cordner AP, Armstrong PJ, Newman SJ, Novo R, Sharkey LC, Jessen EC. Effect of pancreatic tissue sampling on serum pancreatic enzyme levels in clinically healthy dogs. J Vet Diagn Invest. 2010;22:702-7.

13. Pratschke KM, Ryan J, McAlinden A, McLauchlan G. Pancreatic surgical biopsy in 24 dogs and 19 cats: postoperative complications and clinical relevance of histological findings. J Small Anim Pract. 2015;56:60-6.

14. Trivedi S, Marks SL, Kass PH, Luff JA, Keller SM, Johnson EG, et al. Sensitivity and specificity of canine pancreas-specific lipase (cpl) and other markers for pancreatitis in 70 dogs with and without histopathologic evidence of pancreatitis. J Vet Intern Med. 2011;25:1241-7.

15. Steiner JM. Thearpie der akuten pankreatitis beim hund. Tierarztl Prax Ausg K Kleintiere Heimtiere. 2011;39:368-73.

16. Mansfield C, Beths T. Management of acute pancreatitis in dogs: a critical appraisal with focus on feeding and analgesia. J Small Anim Pract. 2015;56:27-39.

17. Sorensen HT, Jacobsen J, Norgaard M, Pedersen L, Johnsen SP, Baron JA. Newer cyclo-oxygenase-2 selective inhibitors, other non-steroidal antiinflammatory drugs and the risk of acute pancreatitis. Aliment Pharmacol Ther. 2006;24:111-6.

18. Blomgren KB, Sundstrom A, Steineck G, Genell S, Sjostedt S, Wiholm BE. A swedish case-control network for studies of drug-induced morbidityacute pancreatitis. Eur J Clin Pharmacol. 2002:58:275-83.

19. Watson P. Pancreatitis in the dog: dealing with a spectrum of disease. Pract. 2004;26:64-77.

20. Van den Bossche I, Paepe D, Saunders J, Hesta M, Daminet S. Acute pancreatitis in dogs and cats: medical imaging, biopsy, treatment and prognosis. Vlaams Diergeneeskd Tijdschr. 2010;79:99-108.

21. Sabre A, Guthrie MM, Maleknia R. Acute necrotising pancreatitis derived from low-dose corticosteroid use: An important reminder of clinical management. BMJ Case Rep. 2015. https://doi.org/10.1136/bcr-2015-209325.

22. Yahiaoui N, Roche M, Aissaoui-Hoffmann N, Keita BA, Mallaret M. Intravenous methylprednisolone induced acute pancreatitis. Eur J Clin Pharmacol. 2017:73:645-6.

23. Mawby DI, Whittemore JC, Fecteau KA. Canine pancreatic-specific lipase concentrations in clinically healthy dogs and dogs with naturally occurring hyperadrenocorticism. J Vet Intern Med. 2014;28:1244-50.

24. Granger LA, Hilferty M, Francis T, Steiner JM, Gaschen L. Variability in the ultrasonographic appearance of the pancreas in healthy dogs compared to dogs with hyperadrenocorticism. Vet Radiol Ultrasound. 2015:56:540-8.

25. Hess RS, Kass PH, Shofer FS, Van Winkle TJ, Washabau RJ. Evaluation of risk factors for fatal acute pancreatitis in dogs. J Am Vet Med Assoc. 1999:214:46-51.

26. Ohta H, Kojima K, Yokoyama N, Sasaki N, Kagawa Y, Hanazono K, et al. Effects of immunosuppressive prednisolone therapy on pancreatic tissue and concentration of canine pancreatic lipase immunoreactivity in healthy dogs. Can J Vet Res. 2018;82:278-86.

27. Tricco AC, Lillie E, Zarin W, O'Brien KK, Colquhoun H, Levac D, et al. Prisma extension for scoping reviews (prisma-scr): Checklist and explanation. Ann Intern Med. 2018;169:467-73.

28. Moher D, Liberati A, Tetzlaff J, Altman DG, PRISMA Group. Preferred reporting items for systematic reviews and meta-analyses: the prisma statement. J Clin Epidemiol. 2009;62:1006-12.

29. Meyer A, Coffey MJ, Oliver MR, Ooi CY. Contrasts and comparisons between childhood and adult onset acute pancreatitis. Pancreatology. 2013:13:429-35.

30. The Centre for Clinical Effectiveness SH, Melbourne „Australia., Evidence-based answers to clinical questions for busy clinicians, 2009. http://www.southernhealth.org.au/icms_docs/2145_EBP_workbook. pdf Accessed Sep 252019

31. Higgins JP, Altman DG, Gotzsche PC, Juni P, Moher D, Oxman AD, et al. The cochrane collaboration's tool for assessing risk of bias in randomised trials. BMJ. 2011;343:d5928.

32. Olivry T, Mueller RS. International Task Force on Canine Atopic Dermatitis. Evidence-based veterinary dermatology: a systematic review of the pharmacotherapy of canine atopic dermatitis. Vet Dermatol. 2003:14:121-46.

33. Summers JF, Brodbelt DC, Forsythe PJ, Loeffler A, Hendricks A The effectiveness of systemic antimicrobial treatment in canine superficial and deep pyoderma: a systematic review. Vet Dermatol. 2012:23:305-29.

34. Jessen LR, Sorensen TM, Bjornvad CR, Nielsen SS, Guardabassi L. Effect of antibiotic treatment in canine and feline urinary tract infections: a systematic review. Vet J. 2015;203:270-7.

35. Jensen AP, Bjornvad CR. Clinical effect of probiotics in prevention or treatment of gastrointestinal disease in dogs: a systematic review. J Vet Intern Med. 2019:33(5):1849-64.

36. Wang ZF, Pan CE, Lu Y, Liu SG, Zhang GJ, Zhang XB. The role of inflammatory mediators in severe acute pancreatitis and regulation of glucocorticoids. Hepatobiliary Pancreat Dis Int. 2003:2:458-62.

37. Eklund A, Leppaniemi A, Kemppainen E, Pettila V. Vasodilatory shock in severe acute pancreatitis without sepsis: Is there any place for hydrocortisone treatment? Acta Anaesthesiol Scand. 2005;49:379-84.

38. Wan MH, Li J, Gong HL, Xue P, Zhu L, Chen GY, et al. Clinical observation on the effect of dexamethasone and chinese herbal decoction for purgation in severe acute pancreatitis patients. Chin J Integr Med. 2011;17:141-5.

39. Studley JG, Schenk WG Jr. Pathophysiology of acute pancreatitis: evaluation of the effect and mode of action of steroids in experimental pancreatitis in dogs. Am J Surg. 1982;143:761-4.

40. Liu X, Liu Q, Pan C. Early preventive treatment for severe acute pancreatitis combined with lung injury. Chin J Traumatol. 2002;5:190-2.

41. Zhang XP, Zhang L, Wang Y, Cheng QH, Wang JM, Cai W, et al. Study of the protective effects of dexamethasone on multiple organ injury in rats with severe acute pancreatitis. JOP. 2007:8:400-12.

42. Schulz HU, Hoenl H, Schrader T, Kropf S, Halangk W, Ochmann C, et al. Randomized, placebo-controlled trial of lazaroid effects on severe acute pancreatitis in rats. Crit Care Med. 2001;29:861-9.

43. Cosen-Binker LI, Binker MG, Negri G, Tiscornia O. Experimental model of acute pancreatitis in wistar rat: glucocorticoid treatment profile. Dig Dis Sci. 2003;48:1453-64

44. Cosen-Binker LI, Binker MG, Cosen R, Negri G, Tiscornia O. Influence of hydrocortisone, prednisolone, and no association on the evolution of acute pancreatitis. Dig Dis Sci. 2006;51:915-25.

45. Jha RK, Ma Q, Sha H, Palikhe M. Protective effect of resveratrol in severe acute pancreatitis-induced brain injury. Pancreas. 2009;38:947-53.

46. Melo CM, Carvalho KM, Neves JC, Morais TC, Rao VS, Santos FA, et al. Alpha, beta-amyrin, a natural triterpenoid ameliorates I-arginine-induced acute pancreatitis in rats. World J Gastroenterol. 2010;16:4272-80. 
47. Ramudo L, Yubero S, Manso MA, Sanchez-Recio J, Weruaga E, De Dios I. Effects of dexamethasone on intercellular adhesion molecule 1 expression and inflammatory response in necrotizing acute pancreatitis in rats. Pancreas. 2010;39:1057-63.

48. Ou JM, Zhang XP, Wu CJ, Wu DJ, Yan P. Effects of dexamethasone and salvia miltiorrhiza on multiple organs in rats with severe acute pancreatitis. J Zhejiang Univ Sci B. 2012;13:919-31.

49. Zhao S, Yang J, Liu T, Zeng J, Mi L, Xiang K. Dexamethasone inhibits nfsmall ka, cyrillicbp65 and hmgb1 expression in the pancreas of rats with severe acute pancreatitis. Mol Med Rep. 2018;18:5345-52.

50. Foitzik T, Forgacs B, Ryschich E, Hotz H, Gebhardt MM, Buhr HJ, et al. Effect of different immunosuppressive agents on acute pancreatitis: a comparative study in an improved animal model. Transplantation. 1998;65:1030-6.

51. Biradar S, Veeresh B. Screening of natural antioxidants by using I-arginine induced acute pancreatitis model. Int J Drug Dev Res. 2012;4:284-97.

52. Biradar S, Veeresh B. Protective effect of lawsone on l-arginine induced acute pancreatitis in rats. Indian J Exp Biol. 2013;51:256-61.

53. Liu C, Dou K, Dou C, Liu J, Zhao Q. Anti-inflammatory effects of tacrolimus in a rat model of acute pancreatitis. Med Chem. 2010;6:37-43.

54. Sha $H, M a ~ Q$, Jha RK, Wang Z. Resveratrol ameliorates lung injury via inhibition of apoptosis in rats with severe acute pancreatitis. Exp Lung Res. 2009;35:344-58.

55. Kilic E, Amanvermez R, Kefeli M, Polat C, Gunay M. Protective effects of etanercept and methylprednisolone on pancreatic damage in ceruleininduced acute pancreatitis. Saudi Med J. 2010;31:394-9.

56. Cui H, Li S, Xu C, Zhang J, Sun Z, Chen H. Emodin alleviates severe acute pancreatitis-associated acute lung injury by decreasing pre-b-cell colony-enhancing factor expression and promoting polymorphonuclear neutrophil apoptosis. Mol Med Rep. 2017;16:5121-8.

57. Okanishi H, Nagata T, Nakane S, Watari T. Comparison of initial treatment with and without corticosteroids for suspected acute pancreatitis in dogs. J Small Anim Pract. 2019;60:298-304.

58. Attix E, Strombeck DR, Wheeldon EB, Stern JS. Effects of an anticholinergic and a corticosteroid on acute pancreatitis in experimental dogs. Am J Vet Res. 1981:42:1668-74

59. Stewart WR, Elliott DW, Zollinger RM. Cortisone in the treatment of experimental acute pancreatitis. Surg Forum. 1958;9:537-41.

60. Imahori SC, Studley JG, Schenk WG Jr. Experimental acute pancreatitis in dogs and effects of steroids. A light and electron microscopic study with reference to pathogenesis. Pathol Res Pract. 1984;178:483-90.

61. Gloor B, UhI W, Tcholakov O, Roggo A, Muller CA, Worni M, et al. Hydrocortisone treatment of early sirs in acute experimental pancreatitis. Dig Dis Sci. 2001;46:2154-61.

62. Ramudo L, Yubero S, Manso MA, Recio JS, Weruaga E, De Dios I. Effect of dexamethasone on peripheral blood leukocyte immune response in bile-pancreatic duct obstruction-induced acute pancreatitis. Steroids. 2010;75:362-7.

63. Duan L, Ma Y, Chi J, Wang X, Wesley AJ, Chen X. The regulatory role of immunosuppressants on immune abnormalities in acute pancreatitis. Biomed Rep. 2014;2:193-8.

64. Zhao Y, Xiong RP, Chen X, Li P, Ning YL, Yang N, et al. Hsp90 regulation affects the treatment of glucocorticoid for pancreatitis-induced lung injury. Mol Cell Biochem. 2018;440:189-97.

65. Kaplan MH, Cotlar AM, Stagg SJ. Acute pancreatitis. Six year survey with evaluation of steroid therapy. Am J Surg. 1964;108:24-30.

66. Wang ZF, Liu C, Lu Y, Dong R, Xu J, Yu L, et al. Dexamethasone and dextran 40 treatment of 32 patients with severe acute pancreatitis. World J Gastroenterol. 2004;10:1333-6.

67. Dong LH, Liu ZM, Wang SJ, Zhao SJ, Zhang D, Chen Y, et al. Corticosteroid therapy for severe acute pancreatitis: a meta-analysis of randomized, controlled trials. Int J Clin Exp Pathol. 2015;8:7654-60.

68. Yu M, Yang Z, Zhu Y, Lu N. Efficacy of glucocorticoids in rodents of severe acute pancreatitis: a meta-analysis. Int J Clin Exp Pathol. 2014;7:3647-61.

69. Christensen MB, Langhorn R, Goddard A, Andreasen EB, Moldal E, Tvarijonaviciute $A$, et al. Comparison of serum amyloid a and c-reactive protein as diagnostic markers of systemic inflammation in dogs. Can Vet J. 2014:55:161-8.
70. Viitanen SJ, Lappalainen AK, Christensen MB, Sankari S, Rajamaki MM. The utility of acute-phase proteins in the assessment of treatment response in dogs with bacterial pneumonia. J Vet Intern Med. 2017;31:124-33.

71. UK Working Party on Acute Pancreatitis. UK guidlines for the management of acute pancreatitis. Gut. 2005:54:1-9.

72. Tenner S, Baillie J, DeWitt J, Vege SS. American College of G. American college of gastroenterology guideline: management of acute pancreatitis. Am J Gastroenterol. 2013;108:1400-15.

73. Holm JL, Rozanski EA, Freeman LM, Webster CRL. C-reactive protein concentrations in canine acute pancreatitis. J Vet Emerg Crit Care. 2004;14:183-6.

74. Sato T, Ohno K, Tamamoto T, Oishi M, Kanemoto H, Fukushima K, et al. Assessment of severity and changes in c-reactive protein concentration and various biomarkers in dogs with pancreatitis. J Vet Med Sci. 2017;79:35-40.

75. De Waele JJ, Hoste EA, Baert D, Hendrickx K, Rijckaert D, Thibo P, et al. Relative adrenal insufficiency in patients with severe acute pancreatitis. Intensive Care Med. 2007;33:1754-60.

76. Peng YS, Wu CS, Chen YC, Lien JM, Tian YC, Fang JT, et al. Critical illnessrelated corticosteroid insufficiency in patients with severe acute biliary pancreatitis: a prospective cohort study. Crit Care. 2009;13:R123.

77. De Waele E, Malbrain M, Spapen HD. How to deal with severe acute pancreatitis in the critically ill. Curr Opin Crit Care. 2019;25:150-6.

78. Burkitt JM, Haskins SC, Nelson RW, Kass PH. Relative adrenal insufficiency in dogs with sepsis. J Vet Intern Med. 2007;21:226-31.

79. Swales H, Batchelor DJ, O'Connell EM. Evaluation of serum cortisol concentration as a prognostic indicator for nonsurvival to hospital discharge in critically ill dogs. J Am Vet Med Assoc. 2020;256:1034-40.

80. Group RC, Horby P, Lim WS, Emberson JR, Mafham M, Bell JL, et al. Dexamethasone in hospitalized patients with covid-19 - preliminary report. $\mathrm{N}$ Engl J Med. 2020. https://doi.org/10.1056/NEJMoa2021436.

81. Janiuk K, Jablonska E, Garley M. Significance of nets formation in covid19. Cells. 2021;10:151.

82. Arcanjo A, Logullo J, Menezes CCB, de Souza Carvalho Giangiarulo TC, Dos Reis MC, de Castro GMM, et al. The emerging role of neutrophil extracellular traps in severe acute respiratory syndrome coronavirus 2 (covid-19). Sci Rep. 2020;10:19630.

83. Li RHL, Tablin F. A comparative review of neutrophil extracellular traps in sepsis. Front Vet Sci. 2018;5:291.

84. Murthy P, Singhi AD, Ross MA, Loughran P, Paragomi P, Papachristou Gl, et al. Enhanced neutrophil extracellular trap formation in acute pancreatitis contributes to disease severity and is reduced by chloroquine. Front Immunol. 2019;10:28.

85. Merza M, Hartman H, Rahman M, Hwaiz R, Zhang E, Renstrom E, et al. Neutrophil extracellular traps induce trypsin activation, inflammation, and tissue damage in mice with severe acute pancreatitis. Gastroenterology. 2015;149:1920-31.

86. Vargas A, Boivin R, Cano P, Murcia Y, Bazin I, Lavoie JP. Neutrophil extracellular traps are downregulated by glucocorticosteroids in lungs in an equine model of asthma. Respir Res. 2017;18:207.

87. Gal Z, Gezsi A, Pallinger E, Visnovitz T, Nagy A, Kiss A, et al. Plasma neutrophil extracellular trap level is modified by disease severity and inhaled corticosteroids in chronic inflammatory lung diseases. Sci Rep. 2020;10:4320

88. Ishihara sangyo kaisha, animal health products. https://www.iskweb.co. jp/eng/products/animal_health.html. Accessed Sep 152020.

89. Moher D, Liberati A, Tetzlaff J, Altman DG, The PRISMA Group (2009). Preferred reporting items for systematic reviews and meta-analyses: the PRISMA Statement. PLoS Med 6(7): e1000097. https://doi.org/10.1371/ journal.pmed.1000097.

\section{Publisher's Note}

Springer Nature remains neutral with regard to jurisdictional claims in published maps and institutional affiliations. 